\title{
OUTCOMES IN PATIENTS WITH PERIPHERAL VASCULAR DISEASE FOLLOWING PERCUTANEOUS CORONARY INTERVENTION
}

John Ramzy ${ }^{a^{*}}$, Nick Andrianopoulos ${ }^{b}$, Louise Roberts ${ }^{c, d}$, Stephen J Duffy, MBBS, PhD ${ }^{\text {b,e }}$, David Clark ${ }^{f}$, Andrew W Teh ${ }^{\mathrm{c}, \mathrm{d}}$, Andrew Ajani ${ }^{\mathrm{a}}$, Christopher M Reid ${ }^{\mathrm{b}, \mathrm{g}}$, Angela Brennan, $\mathrm{RN}^{\mathrm{b}}$, Melanie Freeman ${ }^{\mathrm{c}, \mathrm{d}}$

${ }^{a}$ Department of Cardiology, The Royal Melbourne Hospital, Melbourne, Victoria, Australia

${ }^{b}$ Centre of Cardiovascular Research and Education in Therapeutics (CCRE), Department of Epidemiology and Preventative Medicine, Monash University, Melbourne, Victoria,

Australia

${ }^{c}$ Department of Cardiology, Box Hill Hospital, Melbourne, Victoria, Australia

${ }^{d}$ Monash University Eastern Health Clinical School, Melbourne, Victoria, Australia

${ }^{e}$ Department of Cardiovascular Medicine, Alfred Hospital, Melbourne, Victoria, Australia

${ }^{f}$ Department of Cardiology, Austin Hospital, Melbourne, Victoria, Australia

${ }^{g}$ School of Public Health, Curtin University, Perth, Western Australia

On behalf of the Melbourne Interventional Group (MIG)

* Corresponding Author: Dr John Ramzy, Alfred Heart Centre, $3^{\text {rd }}$ Floor Philip Block, Alfred Hospital, 55 Commercial Rd, Melbourne 3004, AUSTRALIA. Telephone +61 423246960 . E-mail Address: johnramzy@yahoo.com.au

This author takes responsibility for all aspects of the reliability and freedom from bias of the data presented and their discussed interpretation

This is the author manuscript accepted for publication and has undergone full peer review but has not been through the copyediting, typesetting, pagination and proofreading process, which may lead to differences between this version and the Version of Record. Please cite this article as doi: $10.1002 / \operatorname{ccd} .28145$

This article is protected by copyright. All rights reserved. 
The Melbourne Interventional Group (MIG) gratefully acknowledges funding from Abbott, Astra-Zeneca, Medtronic, MSD, Pfizer, Servier, and The Medicines Company. These companies do not have access to data and do not have the right to review manuscripts or abstracts before publication. The MIG Research Group is supported through a National Health \& Medical Research Council of Australia Centre of Research Excellence Grant (No. 1111170) and CMR on a NHMRC Research Fellowship (No. 10458620).

Indexing Words: Coronary Artery Disease, Peripheral Arterial Disease, Revascularisation. Word Count: 4,994

Short Title for running head: Outcomes in Patients with PVD following PCI.

\section{Abstract}

Objectives: To evaluate the clinical characteristics and outcomes of patients with Peripheral Vascular Disease (PVD) undergoing Percutaneous Coronary Intervention (PCI) in a contemporary setting, and to determine whether use of drug-eluting stents (DES) improves outcomes.

Background: PVD was an independent risk factor for adverse outcomes following $\mathrm{PCl}$ in the bare-metal stent (BMS) era. It is not known whether outcomes in these patients have improved with advances in interventional techniques and stent technology, as they have for the general population. 
Methods: 18,380 patients undergoing $\mathrm{PCl}$ from an Australian registry between 2005 and 2013 were studied. Clinical and procedural data, 30-day and 12-month outcomes were compared in those with and without a reported history of PVD. Outcomes were also compared between patients with PVD who received DES and those who received BMS. Long-term mortality was compared using Australian National Death Index linkage.

Results: Patients with PVD ( $n=1,251,6.8 \%)$ were older and had more prevalent diabetes, hypertension, cerebrovascular disease, heart failure, renal impairment, ostial lesions, left main and multi-vessel disease $(p<0.001)$. Patients with PVD had significantly higher rates of major adverse cardiovascular events (MACE) compared with those without PVD, inhospital (5.7\% vs. $4.1 \%, p<0.008)$, at 30 -days (8.6\% vs. $5.8 \%, p<0.001)$ and at 12 -months (24.6\% vs. $13.2 \%, p<0.001)$. At $4.9 \pm 2.6$ years follow-up, there was significantly greater mortality in the PVD group. PVD patients who received DES experienced significantly less MACE than PVD patients treated with BMS at 30-days (4.8 vs. $10.1 \%, p<0.001)$ and $12-$ months (19.4 vs. $26.4 \%, \mathrm{p}<0.005)$.

Conclusions: PVD is an independent predictor of adverse outcomes in patients undergoing PCI. PVD patient who received DES had improved outcomes compared with those receiving BMS. 


\section{Introduction}

The incidence of lower extremity peripheral vascular disease (PVD) has grown by $23.5 \%$ in the first decade of this century. It now affects $3-12 \%$ of the world's population; around 202 million cases worldwide [1]. Patients with PVD have similar atherosclerotic risk profiles to those with coronary artery disease (CAD). In the Global Atherothrombosis Assessment (AGATHA) study, one in two patients with PVD had concomitant CAD, and one in five CAD patients had PVD [2]. Approximately 23\% of patients with both PVD and CAD have undergone percutaneous coronary intervention $(\mathrm{PCl})[3]$. Amongst the overall population of patients undergoing $\mathrm{PCl}$ advanced stent technology and more aggressive adjunctive antiplatelet and anticoagulant therapy have resulted in an improvement in outcomes $[4,5]$. However, there is evidence that this has not been the case for patients with PVD. Despite a reduction in the rate of repeat $\mathrm{PCl}$, their risk of major adverse cardiovascular events outcomes following $\mathrm{PCl}$ has remained unchanged across the early bare-metal stent (BMS) and drug-eluting stent (DES) eras [6]. While PVD is recognized as an independent predictor of poor outcomes after PCI in the BMS era [7-15], there is a paucity of data within the DES era [16]. This study aimed to evaluate whether PVD is an independent predictor of adverse outcomes outcomes in patients undergoing $\mathrm{PCl}$ in a contemporary setting. Furthermore, we aimed to investigate whether patients with PVD represent a subgroup of patients who may derive particular benefit from the use of DES over BMS. 


\section{Methods}

\subsection{Patient Population}

We analysed prospectively collected data from 18,380 consecutive patient procedures in the Melbourne Interventional Group (MIG) registry between 2005 and 2013. No procedures were excluded from the study. All procedures $(n=18,380)$ were divided into two groups. Group one consisted of procedures on patients with a history of PVD $(n=1,251)$ and group two consisted of procedures on patients without PVD $(n=17,129)$. Clinical characteristics, procedural data medication use and in-hospital, 30-day and 12-month adverse outcomes (MACE, death, recurrent myocardial infarction and target vessel revascularisation) were compared between these two groups. We further divided the PVD group into those who had received DES $(n=542)$ and those who received BMS $(n=595)$ and compared 30-day and 12-month adverse outcomes between these two subgroups. Using National Death Index (NDI) linkage, long-term mortality (mean follow-up $4.9 \pm 2.6$ years) was compared between four subgroups: patients without PVD who received DES $(n=7,653)$, patients without PVD who received BMS $(n=8,376)$, patients with PVD who received DES ( $n=539)$ and patients with PVD who received BMS ( $=594)$.

\subsection{Definitions}

Peripheral vascular disease was defined by either a history of chronic or acute occlusion or aneurysmal narrowing of the arterial lumen of the aorta or extremities and includes: claudication with exertion, extremity ischaemic rest pain, amputation for arterial insufficiency, documented aortic aneurysm, documented renal artery stenosis, positive 
non-invasive testing (e.g. ankle brachial index less than 0.9) and vascular reconstruction, bypass surgery or percutaneous intervention to the extremities [17]. Patients were not screened for asymptomatic PVD.

\subsection{Registry Design}

The Melbourne Interventional Group is a collaboration between interventional cardiologists at six major hospitals in Victoria, Australia $[18,19]$. The registry is coordinated by the Centre for Cardiovascular Research \& Education in Therapeutics, a research body within the Department of Epidemiology and Preventative Medicine Monash University, Melbourne, Australia.

The ethics committee of each participating hospital has approved the registry and all participants gave informed consent using an 'opt-out' model. A free-call number that could be used to exclude one's self from the study was provided to patients. Clinical-quality registries have recommended this model [20], which is also used by other large Australian registries [21].

Investigators at each site recorded demographic, clinical and procedural characteristics of all patients undergoing $\mathrm{PCl}$ using a case-report form with standard definitions for all fields [19]. Regular independent audits conducted on 15 verifiable variables from $5 \%$ of MIG procedures have demonstrated $96.5 \%$ accuracy [22], similar to other large registries [23].

\subsection{Procedural decisions}


Taking into account guidelines and clinical factors, the choice between balloon-only angioplasty, $\mathrm{PCl}$ with $\mathrm{BMS}$ or $\mathrm{PCl}$ with $\mathrm{DES}$ was at the discretion of the treating clinician at each centre. In Australian public (government funded) hospitals, DES are currently recommended for patients with lesions at high risk of restenosis including; diabetics, lesions greater than $18 \mathrm{~mm}$ in length, vessels less than $2.5 \mathrm{~mm}$ in diameter, in-stent restenosis and bifurcation or ostial lesions [24]. Peri-procedural antiplatelet and anticoagulant use was generally guideline based, however was also ultimately decided by each patient's treating team.

\subsection{Data Collection and Outcomes}

In-hospital complications were logged at the time of discharge from the index admission. Thirty-day and 12-month follow-up were obtained by telephone contact with the patient, their next-of-kin or treating medical practitioner, and all events were verified by case record review. Long-term mortality was determined using the Australian National Death Index (NDI) linkage, the methodology of which has been previously described in detail [25]. Essentially, The Australian NDI is a database that uses information from the births, deaths and marriages registries of each Australian state to record all deaths nation-wide. Its purpose is to enable epidemiological research. Several demographic variables (name, date of birth, age at death, date of death, gender, state/territory of registration) were used to match deceased patients in the NDI with all patients in the MIG registry. The mean \pm standard deviation NDI follow-up for patients in the MIG registry was $4.9 \pm 2.6$ years.

\subsection{Statistical Analysis}


Continuous variables are expressed as mean \pm standard deviation and categorical as numbers/percentages. The Pearson chi-squared test was used to analyse differences in discrete variables relating to characteristics, procedural data and outcomes between groups. Student's t-test or Kruskal-Wallis equality-of-populations rank test was used for continuous variables as appropriate. The Kaplan-Meier method was used to construct survival curves and the differences in survival were assessed with the log-rank test. A p value of $<0.05$ was considered to indicate statistical significance. Thirty-five baseline clinical and procedural characteristics were considered in the identification of factors independently associated with outcomes at 12-month follow-up. Factors with $p$ value $<0.1$ in simple logistic regression were considered for multiple logistic regression. Statistical analyses were carried out using Stata for Windows (Stata/MP 13.1, College Station, TX, USA). 


\section{Results}

A total of 18,380 patient procedures were enrolled in the registry between 2005 and 2013. Of these, 1,251 patients (6.8\%) had PVD and 17,129 (93.2\%) did not have a history of PVD. DES were used in $44.7 \%$ of procedures overall.

\subsection{Baseline Characteristics}

Patients with PVD were older and had a significantly greater prevalence of cardiovascular risk factors including diabetes, hypertension, dyslipidaemia and a history of $\mathrm{Ml}$ when compared to those without PVD (all p<0.001) (Table 1). They were also more likely to have a history of cerebrovascular disease, chronic pulmonary disease, heart failure, previous $\mathrm{PCl}$ and prior coronary artery bypass grafting (CABG) (all $\mathrm{p}<0.001)$. The PVD group underwent more procedures for elective and non-emergent acute coronary syndromes (ACS) while patients without PVD had a higher rate of $\mathrm{PCl}$ for ST-elevation MI (STEMI) $(p<0.001)$ (Table 1). In keeping with the lower incidence of STEMI in this group, thrombolytics and IIb/IIIa antagonists were used less often in patients with PVD $(p<0.001)$. However, despite the lower incidence of STEMI, the PVD group still had a significant number of patients presenting with out-of-hospital VF-arrest; they also had similar numbers of patients presenting with cardiogenic shock and requiring intra-aortic balloon pump use (Table 1$)$. The proportion of males was similar in both groups $(74.2 \%$ vs. $75.6 \%$, $p=0.25)$ There were more current smokers in the non-PVD group than the PVD group (24.3\% vs. $19.7 \%, p<0.001)$ (Table 1$)$. Comparison of medication use between the two groups (Tables 3 and 4) revealed a significantly greater proportion of non-PVD patients 
were taking statins at 30-days, and beta-blockers and ACE inhibitors at 30-days and 12months. Conversely, the use of ezetimibe, nitrates and calcium channel blockers was significantly higher amongst the PVD group. The PVD group were more often treated with anticoagulation in addition to single or dual-antiplatelet therapy than the non-PVD patients, who had significantly higher rates of dual-antiplatelet therapy alone.

\subsection{Angiographic characteristics}

The procedural success rate was lower in patients with PVD $(94.3 \%$ vs. 95.9\%, p<0.001). They were more likely to have multi-vessel and left main disease than those without PVD ( $76.5 \%$ vs. $58.4 \%, p<0.001$ and $3.4 \%$ vs. $0.8 \%, p<0.001$ respectively) (Table 2). There was a higher rate of in-stent restenosis amongst patients with PVD $(9.5 \%$ vs. $5.8 \%$, $\mathrm{p}<0.001)$ (Table 2). Complex lesions (ACC/AHA B2/C) and ostial lesions were more common amongst the PVD group ( $p=0.013$ and $p<0.001$ respectively), while there was no significant difference in the number of bifurcation lesions $(p=0.075)$ (Table 2$)$. Both DES and BMS use was lower in patients with PVD who had more balloon only procedures than patients without PVD $(p<0.001)$. Detailed angiographic data are shown in Table 2.

\subsection{In-Hospital, 30-Day and 12-Month Clinical Outcomes.}

Patients with PVD experienced more in-hospital MACE (5.7\% vs. 4.1\%, $p=0.008)$, driven by a significantly increased risk of death (3.8\% vs. $2.1 \%, p<0.001)$ (Table 5$)$. They also had higher rates of new renal impairment (1.9\% vs. $1.1 \%, \mathrm{p}=0.015)$, major bleeding $(3.8 \%$ vs. $2.4 \%, p=0.004)$ and peri-procedural arterial dissection at the percutaneous access site (0.6\% vs. $0.2 \%, p=0.003)$ (Table 5). 
There was a higher rate of 30 -day MACE amongst patients with PVD (8.6\% vs. 5.8\%, $\mathrm{p}<0.001)$. Death (5.0\% vs. 2.4\%, p<0.001) and new or recurrent $\mathrm{MI}(3.1 \%$ vs. $2.0 \%$, $p=0.006$ ) occurred significantly more often in the PVD group, while the rate of target vessel revascularisation (TVR) was similar in the two groups (2.2\% vs. 2.4\%, p=0.53) (Table 5).

At 12 months, patients with PVD had higher rates of MACE $(24.6 \%$ vs. $13.2 \%, p<0.001)$, death $(12.2 \%$ vs. $4.2 \%, \mathrm{p}<0.001)$, new or recurrent $\mathrm{MI}(9.8 \%$ vs. $4.4 \%, \mathrm{p}<0.001)$ and TVR (9.5\% vs. $7.2 \%, p=0.003)$ (Table 5).

Independent predictors of MACE at 12 months included PVD (OR 1.35, 95\% Cl: 1.14-1.60, $\mathrm{p}<0.001$ ), cardiogenic shock (OR 5.11, 95\% Cl: 4.14-6.30, p<0.001) and eGFR<30 $\mathrm{ml} / \mathrm{min} / 1.73 \mathrm{~m}^{2}$ (OR 3.09, 95\% Cl: 2.48-3.85, $\left.\mathrm{p}<001\right)$ (Table 6). PVD was also independently associated with 12-month mortality (OR 2.01, 95\% Cl: 1.56-2.59, p<0.001), as were cardiogenic shock and eGFR<30 $\mathrm{ml} / \mathrm{min} / 1.73 \mathrm{~m}^{2}$ (Table 7).

\subsection{PVD and DES Use}

Amongst those with PVD, 542 (43.3\%) received BMS and 595 (47.6\%) received DES with the remaining 114 (9.1\%) patients undergoing balloon angioplasty alone. There was a significantly higher prevalence of diabetes amongst the PVD patients who received DES compared with those who received BMS (54.2\% vs. 35.1\%, p<0.001) (Table 8). PVD patients receiving BMS had a higher rate of STEMI as the indication for $\mathrm{PCI}(24.4 \%$ vs. 9.4\%, p<0.001) while DES was used more often in NSTEMI, unstable angina and nonACS presentations (Table 8). When compared to the PVD patients who received DES, a higher proportion of the PVD patients receiving BMS presented with out of hospital cardiac 
arrest $(2.5 \%$ vs. $0.6 \%, p<0.008)$ and cardiogenic shock (5.9\% vs. $1.9 \%, p<0.001)$ (Table 8$)$. More patients in the PVD and DES group had complex lesions (ACC/AHA classification type B2/C) treated compared to the PVD and BMS group (59.1\% vs. 52.1\%, $\mathrm{p}=0.01)$ (Table 9). As expected, the PVD patients who received DES had higher rates of DAPT use at 30 days and 12 months (95.2\% vs. $89.3 \%, p<0.001$ and $79.8 \%$ vs. $51.4 \%, p<0.001$ respectively) (Tables 10 and 11 ).

Compared with PVD patients who received DES, patients who received BMS had a significantly higher incidence of in-hospital complications including; MACE (6.4\% vs. $2.8 \%$, $\mathrm{p}=0.004)$, death $(4.2 \%$ vs. $1.9 \%, \mathrm{p}=0.022)$ and new or recurrent $\mathrm{MI}(2.0 \%$ vs. $0.2 \%$, $\mathrm{p}=0.004$ ) (Table 12). At 30-days, patients with PVD who received DES had significantly less MACE (4.8\% vs. 10.1\%, p<0.001), death and $\mathrm{MI}$ than those who received BMS. However, the rates of TVR did not differ significantly between the two groups (1.5\% vs. 2.2\%, $p=0.96)$ (Table 12).

At 12-months, there was a significantly lower incidence of MACE $(19.4 \%$ vs. $26.4 \%$, $\mathrm{p}=0.005)$ and death $(8.5 \%$ vs. $14.6 \%, p<0.001)$ amongst patients treated with DES. However, there was no longer a difference in the rate of MI, and TVR continued to be similar (Table 12).

Over a mean follow-up period of $4.9 \pm 2.6$ years patients with PVD who received BMS had the highest mortality (38.4\%) when compared to patients with PVD who received DES (29.1\%) and those without PVD (15.3\% and 12.0\% with BMS and DES respectively, $\mathrm{p}<0.001$ ) (Figure 1). 


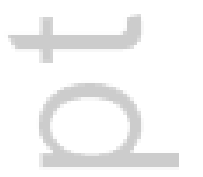

This article is protected by copyright. All rights reserved. 


\section{Discussion}

This is one of the largest registries examining outcomes in patients with PVD following PCI in the DES era. This study has shown that patients with PVD have an increased risk of short-, medium- and long-term adverse outcomes following PCI. Furthermore, PVD is an independent predictor of adverse outcomes following $\mathrm{PCl}$ in the $\mathrm{DES}$ era, despite advances in stent technology and medical therapy. Finally, an important finding of this study is that patients with PVD who were treated with DES had lower rates of death and overall major adverse cardiac events than those who received BMS.

Several studies in the BMS era concluded that PVD was an independent predictor of adverse outcomes after $\mathrm{PCl}[7-11,13-15,26,27]$. Nikolsky et al examined 12-month outcomes in 10,440 consecutive patients undergoing $\mathrm{PCl}$ in the $\mathrm{BMS}$ era, 1,969 of whom had symptomatic lower extremity PVD. The PVD group had a significantly higher 12-month mortality rate $(13.6 \%$ vs. $5.2 \%, \mathrm{p}<0.001)$, and after multivariate analysis, PVD was independently associated with increased 12-month mortality (OR 1.71, 95\% Cl 1.42-2.07, $\mathrm{p}<0.001$ ) [15]. Similarly, Singh et al found that, even after adjustment for concomitant risk factors, patients with PVD treated with $\mathrm{PCl}$ in the $\mathrm{BMS}$ era had an $84 \%$ relative-risk increase in in-hospital mortality and a $48 \%$ relative-risk increase in death at 3 year followup compared to patients without PVD [7]. Analysis of the Tirofiban and Reopro Give Similar Efficacy Outcome Trial (TARGET) showed that PVD was independently associated with a 2 to 3 fold increase in mortality 12 months after $\mathrm{PCl}$ [10]. Amongst patients undergoing $\mathrm{PCl}$ for MI, PVD was independently associated with a doubling of in-hospital 
mortality, and the number of diseased vascular beds in patients with PVD was associated with a graded increase in the risk of adverse outcomes [26].

Similar to findings in the BMS era, our study suggests that PVD continues to be independently associated with roughly a two-fold increased risk of 12-month mortality post$\mathrm{PCl}$ in the DES era.

This study also examined differences in outcomes between patients with PVD who received DES and those PVD patients receiving BMS. Interestingly, we found that patients with PVD who received DES had reduced short-, medium- and long-term MACE and mortality despite no difference in TVR. The difference in mortality was seen out to mean follow-up of $4.9 \pm 2.6$ years. In stent-restenosis and the need for TVR are greatest in the first year after stent insertion and this is when DES have been shown to be superior to BMS in reducing the need for TVR, but not mortality [28]. In the absence of a reduction in TVR, there is no clear explanation for the reduced short- and long-term MACE and mortality in our PVD patients who received DES compared to those who received BMS. However, in this cohort we found a significantly higher rate of $\mathrm{PCl}$ performed for in-stent restenosis in the PVD group. Our data suggests that outcomes for patients with PVD undergoing real world $\mathrm{PCl}$ could possibly be improved by greater use of drug-eluting stents over bare-metal stents in this particular group of patients.

Prior to our registry, only two other studies have compared outcomes between patients with PVD and those without PVD in the DES era $[16,29]$. Midwall et al investigated outcomes amongst 173 patients with PVD and 2,282 patients without a history of PVD who 
underwent $\mathrm{PCl}$ in the early-DES era [16]. The PVD group had significantly higher unadjusted rates of death than the non-PVD group in-hospital $(1.8 \%$ vs. $0.1 \%, p=0.006)$, at 12-months (8.2 vs. 2.9, $\mathrm{p}=0.001)$ and 4 -years (23.8\% vs. $10.8 \%, \mathrm{p}<0.001)$. However, after adjustment for differences in the patient's clinical and angiographic characteristics, PVD was no longer an independent predictor of mortality [16]. In contrast, our study found that PVD remained an independent predictor of adverse outcomes after multivariate adjustment. Of note, the study by Midwall et al had a smaller number of patients and may have been underpowered to detect a true mortality difference after multivariate adjustment. Additionally, the definition of PAD in Midwall's study allowed for the inclusion of patients without PAD of the aorta, renal arteries or extremities, but who had only carotid artery disease or a history of cerebrovascular accident. These patients were not included in our PVD cohort, all of whom had PVD involving the aorta, renal arteries or extremeties. There is some evidence that cerebrovascular disease may be a lesser risk factor for adverse outcomes after $\mathrm{PCl}$ than peripheral arterial disease $[11,14]$ hence their inclusion in the PVD cohort may have reduced the apparent overall increased risk amongst their PVD group.

More recently, an analysis of the Assessment of Dual AntiPlatelet Therapy with Drug Eluting Stents (ADAPT-DES) study was conducted to determine the relationship between PVD, platelet reactivity and subsequent adverse outcomes. There was a $10.2 \%$ prevalence of PVD amongst the 8,582 patients included in the analysis, all of whom received drug-eluting stents. In-line with our findings, PAD was found to be an independent predictor of MACE (adjusted HR 1.34, $\mathrm{p}=0.003$ ), mortality, MI and bleeding at 
two year follow-up. The increased risk amongst PVD patients was not mediated by heightened platelet reactivity (HPR), which affected both PVD and non-PVD patients similarly [29].

The increased rate of adverse outcomes observed in our PVD group may be related to their higher rate of periprocedural bleeding events (3.8\% vs. $2.4 \%, p=0.004)$. Amongst the general population of patients undergoing $\mathrm{PCl}$, periprocedural bleeding has been associated with a 3 to 10-fold increased risk of in-hospital and 30-day mortality [30-34] and a 2 to 4.5-fold increased risk of 12-month mortality [30, 34-37]. Bleeding has also been associated with a greater risk of myocardial infarction and stroke [30, 34]. Analysis of the Harmonizing Outcomes with Revascularization and Stents in Acute Myocardial Infarction (HORIZONS-AMI) trial found an increased risk of MACE and mortality that persisted out to 3 years post-PCI in STEMI patients who had an in-hospital major bleed [38]. Patients with vascular disease may be less able to control the systemic amplification of local clotting mechanisms triggered by bleeding because the counter regulatory pathways are primarily active within endothelial cells. This may predispose them to a hypercoagulable state following a bleeding event [39]. In addition, periprocedural bleeding may lead to antiplatelet or antithrombotic therapy being withheld, which may contribute to the risk of thrombotic complications. Of the patients who experienced bleeding in the Global Registry of Acute Coronary Events (GRACE) registry, there was higher in-hospital mortality in those who had their aspirin, thienopyridine or low-molecular-weight heparin ceased, compared with those in whom the medications were continued in spite of bleeding [40]. Furthermore, 
blood transfusion after $\mathrm{PCl}$ may itself independently increase risk of 12-month mortality $(R R=2.42, p=0.0045)$ amongst patients with severe bleeding [41].

In addition to carrying their own mortality risks, greater age and a higher proportion of comorbidities may influence clinicians to treat residual CAD in these patients less aggressively. Results from the 'Get With the Guidelines' Program that included 37,633 ACS patients with a history of vascular disease found that these patients had a higher adjusted in-hospital mortality than those without a history of vascular disease, yet received less statins, ACE inhibitors and smoking cessation advice [42]. Our analysis suggests that patients with PVD are less often treated with medications that carry prognostic benefit (beta-blockers and ACE-inhibitors) and more often treated with those that portend a symptomatic benefit (nitrates and calcium channel blockers). The increased age and comorbidities among patients with PVD may also negatively impact their participation in exercise-based cardiac rehabilitation, which has been shown to improve medium and longterm mortality in patients after coronary revascularisation [43].

This study highlights the importance of identifying patients with PVD undergoing PCI. Asymptomatic PVD, as detected by non-invasive testing, is 3 to 4 times more common than symptomatic PVD [15]. In a substudy of the Bypass Angioplasty Revascularization (BARI) trial, asymptomatic PVD yielded the same increased mortality risk as symptomatic PVD amongst patients who underwent coronary revascularisation [9]. Seventy per cent of the patients with lower extremity arterial disease in that study would not have been identified without the use of ABI. Reflecting these findings, the 2005 ACCF/AHA guideline for the management of patients with peripheral artery disease was updated in 2011 to 
include a class one recommendation for ankle-brachial index screening for all patients over 65 regardless of symptoms or risk factors [44]. The detection of PVD is important, as it can be a marker of other underlying comorbidities such as CAD and diabetes mellitus that may require intervention.

There were some limitations to this analysis. A registry-based observational study has inherent limitations with data collected prospectively but analysed retrospectively. The reliance on symptomatic reporting and historical evidence in identifying patients with PVD likely resulted in under-recognition of patients with the condition. Finally, patients with severe PVD may have been more likely to be treated medically, thus skewing the population of PVD patients included in our interventional registry.

\section{Conclusion}

PVD is an independent predictor of adverse outcomes following $\mathrm{PCl}$ in the contemporary setting. Further studies are warranted to elucidate the mechanisms responsible for this. The improvement in long-term outcomes seen with drug eluting-stent use was significantly greater for PVD patients than non-PVD patients, suggesting that operators should particularly preference the use of DES over BMS for this high-risk cohort.

\section{References}

[1] Fowkes FG, Rudan D, Rudan I, Aboyans V, Denenberg JO, McDermott MM, et al. Comparison of global estimates of prevalence and risk factors for peripheral artery disease in 2000 and 2010: a systematic review and analysis. Lancet. 2013;382:1329-40. 
[2] Fowkes FG, Low LP, Tuta S, Kozak J, Investigators A. Ankle-brachial index and extent of atherothrombosis in 8891 patients with or at risk of vascular disease: results of the international AGATHA study. European heart journal. 2006;27:1861-7.

[3] Hirsch AT, Criqui MH, Treat-Jacobson D, Regensteiner JG, Creager MA, Olin JW, et al. Peripheral arterial disease detection, awareness, and treatment in primary care. Jama. 2001;286:1317-24.

[4] Singh M, Rihal CS, Berger PB, Bell MR, Grill DE, Garratt KN, et al. Improving outcome over time of percutaneous coronary interventions in unstable angina. Journal of the American College of Cardiology. 2000;36:674-8.

[5] Williams DO, Holubkov R, Yeh W, Bourassa MG, Al-Bassam M, Block PC, et al. Percutaneous coronary intervention in the current era compared with 1985-1986: the National Heart, Lung, and Blood Institute Registries. Circulation. 2000;102:2945-51.

[6] Parikh SV, Saya S, Divanji P, Banerjee S, Selzer F, Abbott JD, et al. Risk of death and myocardial infarction in patients with peripheral arterial disease undergoing percutaneous coronary intervention (from the National Heart, Lung and Blood Institute Dynamic Registry). The American journal of cardiology. 2011;107:959-64.

[7] Singh M, Lennon RJ, Darbar D, Gersh BJ, Holmes DR, Jr., Rihal CS. Effect of peripheral arterial disease in patients undergoing percutaneous coronary intervention with intracoronary stents. Mayo Clinic proceedings. 2004;79:1113-8.

[8] Berger JS, Petersen JL, Brown DL. Vascular disease burden and in-hospital outcomes among patients undergoing percutaneous coronary intervention in New York State. Circulation Cardiovascular interventions. 2009;2:317-22.

[9] Burek KA, Sutton-Tyrrell K, Brooks MM, Naydeck B, Keller N, Sellers MA, et al. Prognostic importance of lower extremity arterial disease in patients undergoing coronary revascularization in the Bypass Angioplasty Revascularization Investigation (BARI). Journal of the American College of Cardiology. 1999;34:716-21.

[10] Chiu JH, Topol EJ, Whitlow PL, Hsu AP, Tuzcu EM, Franco I, et al. Peripheral vascular disease and one-year mortality following percutaneous coronary revascularization. The American journal of cardiology. 2003;92:582-3.

[11] Morikami Y, Natsuaki M, Morimoto T, Ono K, Nakagawa Y, Furukawa Y, et al. Impact of polyvascular disease on clinical outcomes in patients undergoing coronary revascularization: an observation from the CREDO-Kyoto Registry Cohort-2. Atherosclerosis. 2013;228:426-31. [12] Nikolsky E, Mehran R, Dangas GD, Lasic Z, Mintz GS, Negoita M, et al. Prognostic significance of cerebrovascular and peripheral arterial disease in patients having percutaneous coronary interventions. American Journal of Cardiology.93:1536-9. [13] Saw J, Bhatt DL, Moliterno DJ, Brener SJ, Steinhubl SR, Lincoff AM, et al. The influence of peripheral arterial disease on outcomes: a pooled analysis of mortality in eight large randomized percutaneous coronary intervention trials. Journal of the American College of Cardiology. 2006;48:1567-72.

[14] Nikolsky E, Mehran R, Dangas GD, Lasic Z, Mintz GS, Negoita M, et al. Prognostic significance of cerebrovascular and peripheral arterial disease in patients having percutaneous coronary interventions. The American journal of cardiology. 2004;93:1536-9. 
[15] Nikolsky E, Mehran R, Mintz GS, Dangas GD, Lansky AJ, Aymong ED, et al. Impact of symptomatic peripheral arterial disease on 1-year mortality in patients undergoing percutaneous coronary interventions. Journal of endovascular therapy : an official journal of the International Society of Endovascular Specialists. 2004;11:60-70.

[16] Midwall S, Swaminathan RV, Charitakis K, Kim LK, Gordin J, Hriljac I, et al. Impact of peripheral vascular disease on short- and long-term outcomes in patients undergoing nonemergent percutaneous coronary intervention in the drug-eluting stent era. The Journal of invasive cardiology. 2013;25:132-6.

[17] Cannon CP, Battler A, Brindis RG, Cox JL, Ellis SG, Every NR, et al. American College of Cardiology key data elements and definitions for measuring the clinical management and outcomes of patients with acute coronary syndromes. A report of the American College of Cardiology Task Force on Clinical Data Standards (Acute Coronary Syndromes Writing Committee). Journal of the American College of Cardiology. 2001;38:2114-30.

[18] Ajani AE, Szto G, Duffy SJ, Eccleston D, Clark DJ, Lefkovits J, et al. The foundation and launch of the Melbourne Interventional Group: a collaborative interventional cardiology project. Heart, lung \& circulation. 2006;15:44-7.

[19] Chan W, Clark DJ, Ajani AE, Yap CH, Andrianopoulos N, Brennan AL, et al. Progress towards a National Cardiac Procedure Database--development of the Australasian Society of Cardiac and Thoracic Surgeons (ASCTS) and Melbourne Interventional Group (MIG) registries. Heart, lung \& circulation. 2011;20:10-8.

[20] McNeil JJ, Evans SM, Johnson NP, Cameron PA. Clinical-quality registries: their role in quality improvement. The Medical journal of Australia. 2010;192:244-5.

[21] Graves SE, Davidson D, Ingerson L, Ryan P, Griffith EC, McDermott BF, et al. The Australian Orthopaedic Association National Joint Replacement Registry. The Medical journal of Australia. 2004;180:S31-4.

[22] Andrianopoulos N, Dinh D, Duffy SJ, Clark DJ, Brennan AL, Chan W, et al. Quality control activities associated with registries in interventional cardiology and surgery. Heart, lung \& circulation. 2011;20:180-6.

[23] Lagerqvist B, James SK, Stenestrand U, Lindback J, Nilsson T, Wallentin L, et al. Long-term outcomes with drug-eluting stents versus bare-metal stents in Sweden. The New England journal of medicine. 2007;356:1009-19.

[24] Yan BP, Ajani AE, Duffy SJ, New G, Horrigan M, Szto G, et al. Use of drug-eluting stents in Victorian public hospitals. The Medical journal of Australia. 2006;185:363-7.

[25] Wilson WM, Andrianopoulos N, Clark D, Duffy SJ, Brennan A, Harries I, et al. Long-term predictors of mortality after percutaneous coronary intervention in the era of drug-eluting stents. The American journal of cardiology. 2011;108:936-42.

[26] Jeremias A, Gruberg L, Patel J, Connors G, Brown DL. Effect of peripheral arterial disease on in-hospital outcomes after primary percutaneous coronary intervention for acute myocardial infarction. The American journal of cardiology. 2010;105:1268-71.

[27] Guerrero M, Harjai K, Stone GW, Brodie B, Cox D, Boura J, et al. Usefulness of the presence of peripheral vascular disease in predicting mortality in acute myocardial infarction patients treated with primary angioplasty (from the Primary Angioplasty in Myocardial Infarction Database). The American journal of cardiology. 2005;96:649-54. 
[28] Weisz G, Leon MB, Holmes DR, Jr., Kereiakes DJ, Popma JJ, Teirstein PS, et al. Five-year follow-up after sirolimus-eluting stent implantation results of the SIRIUS (Sirolimus-Eluting Stent in De-Novo Native Coronary Lesions) Trial. Journal of the American College of Cardiology. 2009;53:1488-97.

[29] Stone GW, Witzenbichler B, Weisz G, Rinaldi MJ, Neumann FJ, Metzger DC, et al. Platelet reactivity and clinical outcomes after coronary artery implantation of drug-eluting stents (ADAPT-DES): a prospective multicentre registry study. Lancet. 2013;382:614-23. [30] Yatskar L, Selzer F, Feit F, Cohen HA, Jacobs AK, Williams DO, et al. Access site hematoma requiring blood transfusion predicts mortality in patients undergoing percutaneous coronary intervention: data from the National Heart, Lung, and Blood Institute Dynamic Registry. Catheterization and cardiovascular interventions : official journal of the Society for Cardiac Angiography \& Interventions. 2007;69:961-6.

[31] Chhatriwalla AK, Amin AP, Kennedy KF, House JA, Cohen DJ, Rao SV, et al. Association between bleeding events and in-hospital mortality after percutaneous coronary intervention. Jama. 2013;309:1022-9.

[32] Hochholzer W, Wiviott SD, Antman EM, Contant CF, Guo J, Giugliano RP, et al. Predictors of bleeding and time dependence of association of bleeding with mortality: insights from the Trial to Assess Improvement in Therapeutic Outcomes by Optimizing Platelet Inhibition With Prasugrel--Thrombolysis in Myocardial Infarction 38 (TRITON-TIMI 38). Circulation. 2011;123:2681-9.

[33] Kinnaird TD, Stabile E, Mintz GS, Lee CW, Canos DA, Gevorkian N, et al. Incidence, predictors, and prognostic implications of bleeding and blood transfusion following percutaneous coronary interventions. The American journal of cardiology. 2003;92:930-5. [34] Rao SV, Dai D, Subherwal S, Weintraub WS, Brindis RS, Messenger JC, et al. Association between periprocedural bleeding and long-term outcomes following percutaneous coronary intervention in older patients. JACC Cardiovascular interventions. 2012;5:958-65.

[35] Feit F, Voeltz MD, Attubato MJ, Lincoff AM, Chew DP, Bittl JA, et al. Predictors and impact of major hemorrhage on mortality following percutaneous coronary intervention from the REPLACE-2 Trial. The American journal of cardiology. 2007;100:1364-9.

[36] Ndrepepa G, Berger PB, Mehilli J, Seyfarth M, Neumann FJ, Schomig A, et al.

Periprocedural bleeding and 1-year outcome after percutaneous coronary interventions: appropriateness of including bleeding as a component of a quadruple end point. Journal of the American College of Cardiology. 2008;51:690-7.

[37] Wlodarczyk J, Ajani AE, Kemp D, Andrianopoulos N, Brennan AL, Duffy SJ, et al. Incidence, Predictors and Outcomes of Major Bleeding in Patients Following Percutaneous Coronary Interventions in Australia. Heart, lung \& circulation. 2016;25:107-17.

[38] Suh JW, Mehran R, Claessen BE, Xu K, Baber U, Dangas G, et al. Impact of in-hospital major bleeding on late clinical outcomes after primary percutaneous coronary intervention in acute myocardial infarction the HORIZONS-AMI (Harmonizing Outcomes With Revascularization and Stents in Acute Myocardial Infarction) trial. Journal of the American College of Cardiology. 2011;58:1750-6.

[39] Lane DA, Philippou H, Huntington JA. Directing thrombin. Blood. 2005;106:2605-12. 
[40] Spencer FA, Moscucci M, Granger CB, Gore JM, Goldberg RJ, Steg PG, et al. Does comorbidity account for the excess mortality in patients with major bleeding in acute myocardial infarction? Circulation. 2007;116:2793-801.

[41] Kim P, Dixon S, Eisenbrey AB, O'Malley B, Boura J, O'Neill W. Impact of acute blood loss anemia and red blood cell transfusion on mortality after percutaneous coronary intervention. Clinical cardiology. 2007;30:II35-43.

[42] Brilakis ES, Hernandez AF, Dai D, Peterson ED, Banerjee S, Fonarow GC, et al. Quality of care for acute coronary syndrome patients with known atherosclerotic disease: results from the Get With the Guidelines Program. Circulation. 2009;120:560-7.

[43] Heran BS, Chen JM, Ebrahim S, Moxham T, Oldridge N, Rees K, et al. Exercise-based cardiac rehabilitation for coronary heart disease. The Cochrane database of systematic reviews. 2011:CD001800.

[44] Rooke TW, Hirsch AT, Misra S, Sidawy AN, Beckman JA, Findeiss LK, et al. 2011 ACCF/AHA Focused Update of the Guideline for the Management of Patients With Peripheral Artery Disease (updating the 2005 guideline): a report of the American College of Cardiology Foundation/American Heart Association Task Force on Practice Guidelines. Journal of the American College of Cardiology. 2011;58:2020-45.

\section{Figure Legend}

\section{Figure 1}

Long-Term Kaplan-Meier Survival in patients; without Peripheral Vascular Disease (PVD) receiving Drug-eluting Stents (DES), without PVD receiving Bare-Metal Stents (BMS), with PVD receiving DES, and with PVD receiving BMS. 


\begin{tabular}{|c|c|c|c|}
\hline & $\begin{array}{c}\text { PVD } \\
(\mathrm{N}=1,251)\end{array}$ & $\begin{array}{c}\text { No PVD } \\
(\mathrm{N}=17,129)\end{array}$ & $\begin{array}{c}p \\
\text { value }\end{array}$ \\
\hline Mean age - years & $71.0 \pm 10$ & $64 \pm 12$ & $<0.001$ \\
\hline Male gender & $928(74.2 \%)$ & $12,956(75.6 \%)$ & 0.25 \\
\hline \multicolumn{4}{|l|}{ Medical history } \\
\hline Diabetes mellitus & $564(45.1 \%)$ & $3,998(23.4 \%)$ & $<0.001$ \\
\hline Smoking (current) & $244(19.7 \%)$ & $4,137(24.3 \%)$ & $<0.001$ \\
\hline Chronic lung disease & $219(17.6 \%)$ & $1,627(9.5 \%)$ & $<0.001$ \\
\hline Hypertension & $1,088(87.0 \%)$ & $11,206(65.4 \%)$ & $<0.001$ \\
\hline Dyslipidaemia & $1,076(86.1 \%)$ & $11,905(69.6 \%)$ & $<0.001$ \\
\hline Family history of CAD & $464(37.9 \%)$ & 6,596 (38.9\%) & 0.524 \\
\hline Cerebrovascular disease & $282(22.6 \%)$ & $943(5.5 \%)$ & $<0.001$ \\
\hline Heart failure & $154(12.32 \%)$ & $557(3.3 \%)$ & $<0.001$ \\
\hline Previous MI & $608(48.6 \%)$ & $4,495(26.3 \%)$ & $<0.001$ \\
\hline Previous PCl & $441(35.3 \%)$ & $4,221(24.65 \%)$ & $<0.001$ \\
\hline Previous CABG & $311(24.9 \%)$ & $1,308(7.6 \%)$ & $<0.001$ \\
\hline eGFR & & & $<0.001$ \\
\hline$<30$ & $163(13.2 \%)$ & $400(2.4 \%)$ & \\
\hline $30-59$ & $397(32.2 \%)$ & $3,273(19.6 \%)$ & \\
\hline$>60$ & $675(54.7 \%)$ & $13,065(78.1 \%)$ & \\
\hline
\end{tabular}




\begin{tabular}{|c|c|c|c|}
\hline Mean Baseline Serum $\mathrm{Cr}$ & $140.8 \pm 144.7$ & $93.2 \pm 66.3$ & $<0.001$ \\
\hline Mean BMI $\left(\mathrm{kg} / \mathrm{m}^{2}\right)$ & $27.8(27.4-28.1)$ & $28.4(28.3-28.5)$ & $<0.001$ \\
\hline \multicolumn{4}{|l|}{ LVEF } \\
\hline$<30 \%$ & $53(5.0 \%)$ & $350(2.35 \%)$ & \multirow{3}{*}{$<0.001$} \\
\hline $30-45 \%$ & $325(30.8 \%)$ & $3,384(22.7 \%)$ & \\
\hline$>45 \%$ & $677(64.2 \%)$ & $11,172(75.0 \%)$ & \\
\hline \multicolumn{4}{|l|}{ Indication for index $\mathrm{PCl}$} \\
\hline STEMI & $217(17.4 \%)$ & $5,249(30.7 \%)$ & \multirow{4}{*}{$<0.001$} \\
\hline NSTEMI & $413(33.0 \%)$ & $4,503(26.3 \%)$ & \\
\hline Unstable angina & $127(10.2 \%)$ & $1,669(9.8 \%)$ & \\
\hline Non-ACS & $493(39.4 \%)$ & $5,696(33.3 \%)$ & \\
\hline Out of hospital cardiac arrest & $21(1.7 \%)$ & $437(2.6 \%)$ & 0.056 \\
\hline Cardiogenic shock & $52(4.2 \%)$ & $550(3.2 \%)$ & 0.070 \\
\hline IABP & $27(2.2 \%)$ & $422(2.5 \%)$ & 0.50 \\
\hline \multicolumn{4}{|l|}{ Medications } \\
\hline Thrombolytics & $22(1.8 \%)$ & $801(4.7 \%)$ & $<0.001$ \\
\hline IIb/IIla Blockade & $263(21.1 \%)$ & $5,399(31.5 \%)$ & $<0.001$ \\
\hline Heparin & $1229(98.2 \%)$ & $16726(97.7 \%)$ & 0.21 \\
\hline LMWH & $239(19.1 \%)$ & $3272(19.1 \%)$ & 0.99 \\
\hline Bivalirudin & $13(1.1 \%)$ & $258(1.6 \%)$ & 0.15 \\
\hline Aspirin & $1239(99.0 \%)$ & $16900(98.7 \%)$ & 0.28 \\
\hline Clopidogrel & 1167 (93.4\%) & $15918(93.0 \%)$ & 0.55 \\
\hline
\end{tabular}

This article is protected by copyright. All rights reserved. 


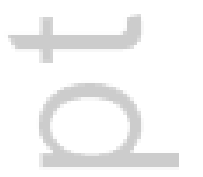

This article is protected by copyright. All rights reserved. 


\begin{tabular}{|c|c|c|c|}
\hline & $\begin{array}{c}\text { PVD } \\
(N=1,251)\end{array}$ & $\begin{array}{c}\text { No PVD } \\
(\mathrm{N}=17,129)\end{array}$ & $\begin{array}{c}\text { p } \\
\text { value }\end{array}$ \\
\hline \multicolumn{4}{|l|}{ Procedure status } \\
\hline Elective & $502(40.3 \%)$ & $5,866(34.3 \%)$ & $<0.001$ \\
\hline Urgent & $739(59.1 \%)$ & $10,985(64.1 \%)$ & \\
\hline Rescue & $10(0.8 \%)$ & $278(1.6 \%)$ & \\
\hline Percutaneous entry location & & & $<0.001$ \\
\hline Femoral & $1,079(86.3 \%)$ & $15,412(90.0 \%)$ & \\
\hline Radial & $137(11.0 \%)$ & $1,671(9.8 \%)$ & \\
\hline Disease extent & & & $<0.001$ \\
\hline Single-vessel disease & $293(23.5 \%)$ & $7,086(41.6 \%)$ & \\
\hline Multi-vessel disease & $954(76.5 \%)$ & $9,963(58.4 \%)$ & \\
\hline \multicolumn{4}{|l|}{ Culprit vessel } \\
\hline Left main coronary artery & $51(3.4 \%)$ & $170(0.8 \%)$ & $<0.001$ \\
\hline Proximal LAD & $193(12.7 \%)$ & $3,302(16.3 \%)$ & $<0.001$ \\
\hline LAD & $415(27.4 \%)$ & $7,057(34.8 \%)$ & $<0.001$ \\
\hline LCX & $228(15.1 \%)$ & $2,668(13.2 \%)$ & 0.036 \\
\hline $\mathrm{RCA}$ & $480(31.7 \%)$ & $6,496(32.1 \%)$ & 0.77 \\
\hline Grafts & $145(9.6 \%)$ & $491(2.4 \%)$ & $<0.001$ \\
\hline Type of coronary lesion & & & $<0.001$ \\
\hline
\end{tabular}




\begin{tabular}{|c|c|c|c|}
\hline De novo & $1,362(89.9 \%)$ & $19,034(93.9 \%)$ & \\
\hline Restenosis (no prior stent) & $9(0.6 \%)$ & $66(0.3 \%)$ & \\
\hline In stent restenosis & $144(9.5 \%)$ & 1,165 (5.8\%) & \\
\hline $\begin{array}{l}\text { Mean no of } \quad \text { lesions } \\
\text { treated/patient }\end{array}$ & $1.2 \pm 0.5$ & $1.2 \pm 0.4$ & 0.02 \\
\hline $\begin{array}{l}\text { Mean no. of stents } \\
\text { deployed/procedure }\end{array}$ & $1.2 \pm 0.7$ & $1.2 \pm 0.6$ & 0.90 \\
\hline Lesion type & & & \\
\hline $\mathrm{B} 2$ and $\mathrm{C}$ & $869(57.4 \%)$ & $10,960(54.1 \%)$ & 0.013 \\
\hline Mean stent diameter (mm\%) & $2.9 \pm 0.5$ & $2.9 \pm 0.5$ & 0.20 \\
\hline Mean stent length (mm\%) & $16.9 \pm 5.3$ & $17.3 \pm 5.6$ & 0.009 \\
\hline Bifurcation lesion & $142(9.4 \%)$ & $2,197(10.8 \%)$ & 0.075 \\
\hline Ostial lesion & $142(9.4 \%)$ & $1,368(6.8 \%)$ & $<0.001$ \\
\hline \multicolumn{4}{|l|}{ Type of stent } \\
\hline Balloon only & $114(9.1 \%)$ & $1,066(6.2 \%)$ & $<0.001$ \\
\hline Bare metal & $595(47.6 \%)$ & $8,386(49.0 \%)$ & \\
\hline Drug eluting & $542(43.3 \%)$ & $7,677(44.8 \%)$ & \\
\hline Procedural success rate & $1357(94.3 \%)$ & $17,846(95.9 \%)$ & 0.004 \\
\hline
\end{tabular}




\begin{tabular}{|c|c|c|c|}
\hline & $\begin{array}{c}\text { PVD } \\
(N=1,090)\end{array}$ & $\begin{array}{c}\text { No PVD } \\
(\mathrm{N}=15,601)\end{array}$ & $\begin{array}{c}p \\
\text { value }\end{array}$ \\
\hline \multicolumn{4}{|l|}{ Antiplatelet/Anticoagulant } \\
\hline No AP and no AC & $4(0.4 \%)$ & $34(0.2 \%)$ & 0.318 \\
\hline Aspirin only & $52(4.8 \%)$ & $593(3.8 \%)$ & 0.108 \\
\hline Thienopyridine only & $22(2.0 \%)$ & $193(1.2 \%)$ & 0.027 \\
\hline Aspirin and Thienopyridine & $985(89.6 \%)$ & $14,673(93.2 \%)$ & $<0.001$ \\
\hline Anticoagulation only & $6(0.6 \%)$ & $19(0.1 \%)$ & $<0.001$ \\
\hline Single AP and AC & $30(2.8 \%)$ & $213(1.4 \%)$ & $<0.001$ \\
\hline Triple therapy & $78(7.2 \%)$ & $813(5.2 \%)$ & 0.006 \\
\hline Statin & $998(91.6 \%)$ & $14,816(94.7)$ & $<0.001$ \\
\hline Fibrate & $24(2.6 \%)$ & $224(1.8 \%)$ & 0.070 \\
\hline Ezetimibe & $100(10.8 \%)$ & $586(4.6 \%)$ & $<0.001$ \\
\hline Beta Blocker & $774(71.3 \%)$ & $12,142(77.9 \%)$ & $<0.001$ \\
\hline ACE inhibitor & $599(55.3 \%)$ & $9,942(63.8 \%)$ & $<0.001$ \\
\hline ARB & $287(26.5 \%)$ & $2,722(17.5 \%)$ & $<0.001$ \\
\hline Nitrate & $200(21.8 \%)$ & 1, $056(8.4 \%)$ & $<0.001$ \\
\hline Ca Channel Blocker & $268(29.1 \%)$ & 1, 975 (15.7) & $<0.001$ \\
\hline
\end{tabular}




\begin{tabular}{|c|c|c|c|}
\hline & $\begin{array}{c}\text { PVD } \\
(\mathrm{N}=966)\end{array}$ & $\begin{array}{c}\text { No PVD } \\
(\mathrm{N}=14,696)\end{array}$ & $\begin{array}{c}p \\
\text { value }\end{array}$ \\
\hline \multicolumn{4}{|l|}{ Antiplatelet/Anticoagulant } \\
\hline No AP and no AC & $13(1.4 \%)$ & $167(1.1 \%)$ & 0.528 \\
\hline Aspirin only & $231(23.9 \%)$ & $3,890(26.1 \%)$ & 0.121 \\
\hline Thienopyridine only & $39(4.0 \%)$ & $493(3.3 \%)$ & 0.230 \\
\hline Aspirin and Thienopyridine & $619(63.7 \%)$ & $9,785(65.6 \%)$ & 0.233 \\
\hline Anticoagulation only & $14(1.5 \%)$ & $47(0.3 \%)$ & 0.000 \\
\hline Single $A P$ and $A C$ & $52(5.4 \%)$ & $520(3.5 \%)$ & 0.002 \\
\hline Triple therapy & $34(3.5 \%)$ & $377(2.5 \%)$ & 0.006 \\
\hline Statin & $882(91.2 \%)$ & $13,828(92.7)$ & 0.079 \\
\hline Fibrate & $25(2.7 \%)$ & $291(2.1 \%)$ & 0.211 \\
\hline Ezetimibe & $114(12.4 \%)$ & $943(6.8 \%)$ & $<0.001$ \\
\hline Beta Blocker & $630(65.8 \%)$ & 10, $564(71.3 \%)$ & $<0.001$ \\
\hline ACE inhibitor & $490(51.3 \%)$ & $8,718(58.9 \%)$ & $<0.001$ \\
\hline ARB & $276(28.8 \%)$ & $3,154(21.3 \%)$ & $<0.001$ \\
\hline Nitrate & $153(16.7 \%)$ & $1,160(8.4 \%)$ & $<0.001$ \\
\hline Ca Channel Blocker & $302(33.2 \%)$ & 2, 406 (17.5) & $<0.001$ \\
\hline
\end{tabular}


Table 5. In-Hospital, 30-Day and 12-Month Clinical Outcomes

\begin{tabular}{|c|c|c|c|}
\hline & $\begin{array}{c}\text { PVD } \\
(N=1,251)\end{array}$ & $\begin{array}{c}\text { No PVD } \\
(\mathrm{N}=17,129)\end{array}$ & $p$ value \\
\hline \multicolumn{4}{|l|}{ In-Hospital } \\
\hline MACE & $71(5.7 \%)$ & $705(4.1 \%)$ & 0.008 \\
\hline Death & $47(3.8 \%)$ & $357(2.1 \%)$ & $<0.001$ \\
\hline New or recurrent $\mathrm{MI}$ & $17(1.4 \%)$ & $199(1.2 \%)$ & 0.534 \\
\hline Repeat PCl & $3(0.2 \%)$ & $108(0.6 \%)$ & 0.084 \\
\hline CABGs & $10(0.8 \%)$ & $148(0.9 \%)$ & 0.806 \\
\hline New Renal Impairment & $24(1.9 \%)$ & $196(1.1 \%)$ & 0.015 \\
\hline Bleeding event & $47(3.8 \%)$ & $418(2.4 \%)$ & 0.004 \\
\hline Site of bleeding & & & 0.698 \\
\hline Retroperitoneal & $4(8.7 \%)$ & $24(6 \%)$ & \\
\hline Percutaneous entry site & $16(34.8 \%)$ & $157(39.4 \%)$ & \\
\hline Other & $26(56.5 \%)$ & $218(54.6 \%)$ & \\
\hline \multicolumn{4}{|l|}{ Vascular Complication } \\
\hline Access Site Occlusion & 0 & $6(0.04 \%)$ & 0.501 \\
\hline Loss of distal pulse & $1(0.1 \%)$ & $17(0.1 \%)$ & 0.833 \\
\hline Peripheral Arterial Dissection & $7(0.6 \%)$ & $29(0.2 \%)$ & 0.003 \\
\hline AV Fistula & $1(0.1 \%)$ & $9(0.1 \%)$ & 0.688 \\
\hline Pseudoaneurysm & $1(0.1 \%)$ & $56(0.3 \%)$ & 0.129 \\
\hline
\end{tabular}




\begin{tabular}{lccc}
\hline 30-Days & & & \\
MACE & $107(8.6 \%)$ & $996(5.8 \%)$ & $<0.001$ \\
Death & $62(5.0 \%)$ & $416(2.4 \%)$ & $<0.001$ \\
\multicolumn{1}{c}{ Cardiac cause } & $48(77.4 \%)$ & $329(79.3 \%)$ & 0.737 \\
Myocardial infarction & $39(3.12 \%)$ & $339(2.0 \%)$ & 0.006 \\
TVR & $27(2.2 \%)$ & $418(2.4 \%)$ & 0.531 \\
\hline 12-Months & $308(24.6 \%)$ & $2264(13.2 \%)$ & $<0.001$ \\
MACE & $153(12.2 \%)$ & $720(4.2 \%)$ & $<0.001$ \\
Death & $90(58.8 \%)$ & $431(60.1 \%)$ & 0.768 \\
\multicolumn{1}{c}{ Cardiac cause } & $123(9.8 \%)$ & $758(4.4 \%)$ & $<0.001$ \\
Myocardial infarction & $119(9.5 \%)$ & $1236(7.2 \%)$ & 0.003 \\
TVR & & & \\
\hline
\end{tabular}


Table 6. Predictors of 12-Month MACE

\begin{tabular}{|c|c|c|c|}
\hline Variable & OR & $95 \% \mathrm{Cl}$ & $p$ value \\
\hline PVD & 1.35 & $1.14-1.60$ & $<0.001$ \\
\hline Age (per year) & 1.01 & $1.00-1.01$ & $<0.001$ \\
\hline Diabetes & 1.38 & $1.23-1.54$ & $<0.001$ \\
\hline PHx CABGs & 1.47 & $1.15-1.89$ & 0.002 \\
\hline Hypertension & 1.29 & $1.14-1.44$ & $<0.001$ \\
\hline Reference vessel $<2.5 \mathrm{~mm}$ & 1.45 & $1.29-1.62$ & $<0.001$ \\
\hline LAD lesion & 1.19 & $1.07-1.32$ & 0.001 \\
\hline $\mathrm{PHx} \mathrm{MI}$ & 1.24 & $1.12-1.39$ & $<0.001$ \\
\hline B2/C lesion & 1.52 & $1.37-1.69$ & $<0.001$ \\
\hline Ostial lesion & 1.38 & $1.17-1.63$ & $<0.001$ \\
\hline \multicolumn{4}{|l|}{ eGFR } \\
\hline$<30$ & 3.09 & $2.48-3.85$ & $<0.001$ \\
\hline $30-59$ & 1.21 & $1.08-1.37$ & 0.002 \\
\hline \multicolumn{4}{|l|}{ LVEF } \\
\hline$<30 \%$ & 2.61 & $2.05-3.32$ & $<0.001$ \\
\hline $30-45 \%$ & 1.44 & $1.29-1.60$ & $<0.001$ \\
\hline Cardiogenic shock & 5.11 & $4.14-6.30$ & $<0.001$ \\
\hline Thrombolysis & 0.68 & $0.52-0.88$ & 0.003 \\
\hline DES & 0.48 & $0.43-0.53$ & $<0.001$ \\
\hline
\end{tabular}




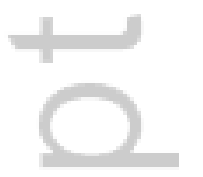

This article is protected by copyright. All rights reserved. 
Table 7. Predictors of 12-Month Mortality

\begin{tabular}{|c|c|c|c|}
\hline Variable & OR & $95 \% \mathrm{Cl}$ & $p$ value \\
\hline PVD & 2.01 & $1.56-2.59$ & $<0.001$ \\
\hline Age & 1.04 & $1.03-1.05$ & $<0.001$ \\
\hline MVD & 1.29 & $1.06-1.58$ & 0.013 \\
\hline Diabetes & 1.44 & $1.19-1.75$ & $<0.001$ \\
\hline Urgent or Rescue $\mathrm{PCl}$ & 2.23 & $1.72-2.88$ & $<0.001$ \\
\hline CLD & 1.58 & $1.25-2.01$ & $<0.001$ \\
\hline Dyslipidaemia & 0.67 & $0.55-0.81$ & $<0.001$ \\
\hline$C D$ & 1.40 & $1.07-1.84$ & 0.014 \\
\hline DES & 0.66 & $0.54-0.80$ & $<0.001$ \\
\hline B2/C lesion & 1.39 & $1.14-1.69$ & 0.001 \\
\hline \multicolumn{4}{|l|}{ eGFR } \\
\hline$<30$ & 5.44 & $4.03-7.34$ & $<0.001$ \\
\hline $30-59$ & 1.85 & $1.51-2.26$ & $<0.001$ \\
\hline \multicolumn{4}{|l|}{ LVEF } \\
\hline$<30 \%$ & 5.23 & $3.83-7.14$ & $<0.001$ \\
\hline $30-45 \%$ & 1.82 & $1.50-2.20$ & $<0.001$ \\
\hline
\end{tabular}




\begin{tabular}{lccc}
\hline Reference vessel $<2.5 \mathrm{~mm}$ & 1.35 & $1.10-1.65$ & 0.003 \\
Cardiogenic shock & 9.18 & $7.14-11.80$ & $<0.001$ \\
RCA lesion & 0.70 & $0.57-0.86$ & 0.001 \\
\hline
\end{tabular}

Table 8. Baseline characteristics in PVD patients with BMS vs. DES

\begin{tabular}{|c|c|c|c|}
\hline & $\begin{array}{l}\text { PVD + BMS } \\
(N=595)\end{array}$ & $\begin{array}{c}\text { PVD + DES } \\
(N=542)\end{array}$ & $\begin{array}{c}\text { p } \\
\text { value }\end{array}$ \\
\hline Mean age - years & $72 \pm 11$ & $70 \pm 10$ & $<0.001$ \\
\hline Male gender & $442(74.3 \%)$ & $402(74.2 \%)$ & 0.96 \\
\hline \multicolumn{4}{|l|}{ Medical history } \\
\hline Diabetes mellitus & $209(35.1 \%)$ & $294(54.2 \%)$ & $<0.001$ \\
\hline Smoking (current) & $112(19.0 \%)$ & $121(22.5 \%)$ & 0.134 \\
\hline Chronic lung disease & $121(20.4 \%)$ & $83(15.4 \%)$ & 0.029 \\
\hline Hypertension & $501(84.3 \%)$ & $486(89.7 \%)$ & 0.008 \\
\hline Dyslipidaemia & $496(83.4 \%)$ & $479(88.5 \%)$ & 0.012 \\
\hline Family history of CAD & $201(34.5 \%)$ & $218(41.1 \%)$ & 0.023 \\
\hline Cerebrovascular disease & $139(23.4 \%)$ & $117(21.6 \%)$ & 0.465 \\
\hline Heart failure & $71(12.0 \%)$ & $65(12.0 \%)$ & 0.984 \\
\hline Previous MI & $258(43.4 \%)$ & $273(50.4 \%)$ & 0.018 \\
\hline Previous $\mathrm{PCl}$ & $161(27.1 \%)$ & $221(40.8 \%)$ & $<0.001$ \\
\hline Previous CABG & $118(19.8 \%)$ & $149(27.5 \%)$ & 0.002 \\
\hline
\end{tabular}




\begin{tabular}{|c|c|c|c|}
\hline eGFR & & & 0.060 \\
\hline$<30$ & $73(12.5 \%)$ & $71(13.2 \%)$ & \\
\hline $30-59$ & $207(35.4 \%)$ & $155(28.8 \%)$ & \\
\hline$>60$ & 305 (52.1\%) & $312(58.0 \%)$ & \\
\hline Mean Baseline Serum Cr & $138.6 \pm 135.8$ & $138.3 \pm 142.5$ & 0.969 \\
\hline Mean BMI $\left(\mathrm{kg} / \mathrm{m}^{2}\right)$ & $27.3(26.9-27.8)$ & $28.2(27.7-28.7)$ & 0.009 \\
\hline \multicolumn{4}{|l|}{ LVEF } \\
\hline$<30 \%$ & $25(5.0 \%)$ & $23(4.9 \%)$ & 0.437 \\
\hline $30-45 \%$ & $159(32.1 \%)$ & $132(28.3 \%)$ & \\
\hline$>45 \%$ & $312(62.9 \%)$ & $311(66.7 \%)$ & \\
\hline \multicolumn{4}{|l|}{ Indication for index $\mathrm{PCl}$} \\
\hline STEMI & $145(24.4 \%)$ & $51(9.4 \%)$ & \\
\hline NSTEMI & $183(30.8 \%)$ & $201(37.1 \%)$ & \\
\hline Unstable angina & $51(8.6 \%)$ & $65(12.0 \%)$ & \\
\hline Non-ACS & $215(36.2 \%)$ & $225(41.5 \%)$ & \\
\hline Out of hospital cardiac arrest & $15(2.5 \%)$ & $3(0.6 \%)$ & 0.008 \\
\hline Cardiogenic shock & $35(5.9 \%)$ & $10(1.9 \%)$ & $<0.001$ \\
\hline IABP & $19(3.2 \%)$ & $5(0.9 \%)$ & 0.008 \\
\hline \multicolumn{4}{|l|}{ Medications } \\
\hline Thrombolytics & $15(2.5 \%)$ & $6(1.1 \%)$ & 0.077 \\
\hline Ilb/IIla Blockade & 139 (23.4\%) & $104(19.3 \%)$ & 0.096 \\
\hline Heparin & $586(98.5 \%)$ & $530(97.8 \%)$ & 0.380 \\
\hline
\end{tabular}




\begin{tabular}{lccc}
\hline LMWH & $120(20.2 \%)$ & $101(18.7 \%)$ & 0.524 \\
Bivalirudin & $9(1.6 \%)$ & $3(0.6 \%)$ & 0.116 \\
Aspirin & $590(99.2 \%)$ & $535(98.7 \%)$ & 0.457 \\
Clopidogrel & $573(96.5 \%)$ & $518(95.8 \%)$ & 0.533 \\
Ticagrelor & $10(1.7 \%)$ & $16(3.0 \%)$ & 0.408 \\
Prasugrel & $9(1.5 \%)$ & $12(2.2 \%)$ & 0.652 \\
\hline
\end{tabular}


Table 9. Angiographic characteristics in PVD patients with BMS vS. DES

\begin{tabular}{|c|c|c|c|}
\hline & $\begin{array}{c}\text { PVD + BMS } \\
(N=595)\end{array}$ & $\begin{array}{l}\text { PVD + DES } \\
(N=542)\end{array}$ & $\begin{array}{c}p \\
\text { value }\end{array}$ \\
\hline \multicolumn{4}{|l|}{ Procedure status } \\
\hline Elective & $217(36.5 \%)$ & $232(42.8 \%)$ & 0.056 \\
\hline Urgent & $375(63.0 \%)$ & $305(56.3 \%)$ & \\
\hline Rescue & $3(0.5 \%)$ & $5(0.9 \%)$ & \\
\hline Percutaneous entry location & & & 0.890 \\
\hline Femoral & $512(86.1 \%)$ & $469(86.5 \%)$ & \\
\hline Radial & $66(11.1 \%)$ & $60(11.1 \%)$ & \\
\hline Disease extent & & & 0.040 \\
\hline Single-vessel disease & $156(26.3 \%)$ & $114(21.1 \%)$ & \\
\hline Multi-vessel disease & $437(73.7 \%)$ & $426(78.9 \%)$ & \\
\hline \multicolumn{4}{|l|}{ Culprit vessel } \\
\hline Left main coronary artery & $17(2.5 \%)$ & $28(4.1 \%)$ & 0.096 \\
\hline Proximal LAD & $105(15.4 \%)$ & $72(10.6 \%)$ & 0.008 \\
\hline LAD & $193(28.3 \%)$ & $188(27.6 \%)$ & 0.750 \\
\hline LCx & $100(14.7 \%)$ & $110(16.1 \%)$ & 0.460 \\
\hline $\mathrm{RCA}$ & $249(36.6 \%)$ & $186(27.3 \%)$ & $<0.001$ \\
\hline Grafts & $54(7.9 \%)$ & $79(11.6 \%)$ & 0.023 \\
\hline Type of coronary lesion & & & $<0.001$ \\
\hline
\end{tabular}




\begin{tabular}{lccc}
\hline De novo & $665(97.7 \%)$ & $585(85.8 \%)$ & \\
Restenosis (no prior stent) & $3(0.4 \%)$ & $4(0.6 \%)$ & \\
In stent restenosis & $13(1.9 \%)$ & $93(13.6 \%)$ & \\
\hline Mean no of lesions & $1.2 \pm 0.4$ & $1.3 \pm 0.5$ & 0.046 \\
treated/patient & & & \\
\hline \multicolumn{1}{c}{ nean of stents } & $1.3 \pm 0.6$ & $1.4 \pm 0.6$ & $<0.001$ \\
deployed/procedure & & & \\
\hline Lesion type & $355(52.1 \%)$ & $403(59.1 \%)$ & 0.010 \\
B2 and C & $3.1 \pm 0.5$ & $2.8 \pm 0.4$ & $<0.001$ \\
\hline Mean stent diameter (mm\%) & $15.7 \pm 4.3$ & $18.1 \pm 6.0$ & $<0.001$ \\
\hline Mean stent length (mm\%) & $48(7.1 \%)$ & $75(11.0 \%)$ & 0.011 \\
\hline Bifurcation lesion & $51(7.5 \%)$ & $70(10.3 \%)$ & 0.072 \\
\hline Ostial lesion & $644(99.4 \%)$ & $636(98.0 \%)$ & 0.028 \\
\hline Procedural success rate & & & \\
\hline
\end{tabular}


Table 10. 30 Day Medication Use in PVD patients with BMS vs. DES

\begin{tabular}{lccc}
\hline & PVD + BMS & PVD + DES & p \\
& (N = 595) & $(\mathbf{N}=542)$ & value \\
\hline Antiplatelet/Anticoagulant & & & \\
No AP and no AC & $3(0.6 \%)$ & $0(0.0 \%)$ & 0.088 \\
Aspirin only & $25(4.9 \%)$ & $4(0.8 \%)$ & $<0.001$ \\
Thienopyridine only & $10(2.0 \%)$ & $10(2.0 \%)$ & 0.939 \\
Aspirin and Thienopyridine & $457(89.3 \%)$ & $472(95.2 \%)$ & $<0.001$ \\
Anticoagulation only & $4(0.8 \%)$ & $1(0.2 \%)$ & 0.191 \\
Single AP and AC & $13(2.6 \%)$ & $9(1.8 \%)$ & 0.434 \\
Triple therapy & $59(11.6 \%)$ & $13(2.6 \%)$ & $<0.001$ \\
\hline Statin & $461(90.8 \%)$ & $453(91.9)$ & 0.523 \\
Fibrate & $17(3.9 \%)$ & $7(1.7 \%)$ & 0.056 \\
Ezetimibe & $36(8.2 \%)$ & $50(12.2 \%)$ & 0.058 \\
Beta Blocker & $351(69.6 \%)$ & $356(72.4 \%)$ & 0.345 \\
ACE inhibitor & $271(54.0 \%)$ & $278(56.5 \%)$ & 0.424 \\
ARB & $130(25.8 \%)$ & $139(28.3 \%)$ & 0.372 \\
Nitrate & $97(22.4 \%)$ & $85(20.7 \%)$ & 0.556 \\
Ca Channel Blocker & $118(27.3 \%)$ & $120(29.3)$ & 0.529 \\
\hline
\end{tabular}


Table 11. 12-Month Medication Use in PVD patients with BMS vs. DES

\begin{tabular}{lccc}
\hline & PVD + BMS & PVD + DES & p \\
& (N = 595) & (N = 542) & value \\
\hline Antiplatelet/Anticoagulant & & & \\
No AP and no AC & $10(2.3 \%)$ & $2(0.5 \%)$ & 0.018 \\
Aspirin only & $146(33.3 \%)$ & $57(12.7 \%)$ & $<0.001$ \\
Thienopyridine only & $13(3.0 \%)$ & $21(4.7 \%)$ & 0.183 \\
Aspirin and Thienopyridine & $226(51.4 \%)$ & $360(79.8 \%)$ & $<0.001$ \\
Anticoagulation only & $7(1.6 \%)$ & $3(0.7 \%)$ & 0.191 \\
Single AP and AC & $37(8.5 \%)$ & $6(1.3 \%)$ & $<0.001$ \\
Triple therapy & $22(5.0 \%)$ & $10(2.2 \%)$ & 0.026 \\
\hline Statin & $398(90.7 \%)$ & $407(90.9)$ & 0.923 \\
Fibrate & $17(4.0 \%)$ & $8(1.9 \%)$ & 0.069 \\
Ezetimibe & $41(9.7 \%)$ & $58(13.8 \%)$ & 0.067 \\
Beta Blocker & $280(64.5 \%)$ & $298(67.1 \%)$ & 0.417 \\
ACE inhibitor & $217(49.9 \%)$ & $231(52.3 \%)$ & 0.481 \\
ARB & $126(28.9 \%)$ & $132(29.9 \%)$ & 0.754 \\
Nitrate & $67(16.0 \%)$ & $67(16.0 \%)$ & 1.000 \\
Ca Channel Blocker & $125(29.9 \%)$ & $149(35.7)$ & 0.077 \\
\hline
\end{tabular}


Table 12. In-Hospital, 30-Day and 12-Month Outcomes in PVD patients with BMS vs. DES

\begin{tabular}{lccc}
\hline & PVD + BMS & PVD + DES & p value \\
& (N = 595) & (N = 542) & \\
\hline In-Hospital & $38(6.4 \%)$ & $15(2.8 \%)$ & 0.004 \\
MACE & $25(4.2 \%)$ & $10(1.9 \%)$ & 0.022 \\
Death & $12(2.0 \%)$ & $1(0.2 \%)$ & 0.004 \\
New or recurrent MI & $1(0.2 \%)$ & $2(0.4 \%)$ & 0.507 \\
Repeat PCl & $4(0.7 \%)$ & $1(0.2 \%)$ & 0.215 \\
CABGs & $13(2.2 \%)$ & $6(1.1 \%)$ & 0.158 \\
New Renal Impairment & $22(3.7 \%)$ & $20(3.7 \%)$ & 0.995 \\
Bleeding event & & & 0.721 \\
Site of bleeding & $2(9.5 \%)$ & $2(10.0 \%)$ & \\
$\quad$ Retroperitoneal & $6(28.6 \%)$ & $8(40.0 \%)$ & \\
Percutaneous entry site & $13(61.9 \%)$ & $10(50.0 \%)$ & \\
Other & & & \\
Vascular Complication & 0 & 0 & \\
Access Site Occlusion & & & \\
\hline
\end{tabular}




\begin{tabular}{|c|c|c|c|}
\hline Loss of distal pulse & $0(0.0 \%)$ & $1(0.2 \%)$ & 0.295 \\
\hline Peripheral Arterial Dissection & $4(0.7 \%)$ & $3(0.6 \%)$ & 0.158 \\
\hline AV Fistula & $1(0.2 \%)$ & $0(0.0 \%)$ & 0.340 \\
\hline Pseudoaneurysm & $0(0.0 \%)$ & $1(0.2 \%)$ & 0.295 \\
\hline \multicolumn{4}{|l|}{ 30-Days } \\
\hline MACE & $60(10.1 \%)$ & $26(4.8 \%)$ & 0.001 \\
\hline Death & $35(5.9 \%)$ & $14(2.6 \%)$ & 0.006 \\
\hline Cardiac cause & $25(71.4 \%)$ & $11(78.6 \%)$ & 0.609 \\
\hline Myocardial infarction & $25(4.2 \%)$ & $9(1.7 \%)$ & 0.012 \\
\hline TVR & $13(2.2 \%)$ & $8(1.5 \%)$ & 0.375 \\
\hline \multicolumn{4}{|l|}{ 12-Months } \\
\hline MACE & $157(26.4 \%)$ & $105(19.4 \%)$ & 0.005 \\
\hline Death & $87(14.6 \%)$ & $46(8.5 \%)$ & 0.001 \\
\hline Cardiac cause & $44(50.6 \%)$ & $30(65.2 \%)$ & 0.106 \\
\hline Myocardial infarction & $61(10.3 \%)$ & $44(8.1 \%)$ & 0.214 \\
\hline TVR & $51(8.6 \%)$ & $46(8.5 \%)$ & 0.959 \\
\hline
\end{tabular}




\section{OUTCOMES IN PATIENTS WITH PERIPHERAL VASCULAR DISEASE FOLLOWING PERCUTANEOUS CORONARY INTERVENTION}

John Ramzy ${ }^{a^{*}}$, Nick Andrianopoulos ${ }^{b}$, Louise Roberts ${ }^{c, d}$, Stephen J Duffy, MBBS, PhD ${ }^{\text {b,e }}$, David Clark ${ }^{f}$, Andrew W Teh ${ }^{\mathrm{c}, \mathrm{d}}$, Andrew Ajani $^{\mathrm{a}}$, Christopher M Reid ${ }^{\mathrm{b}, \mathrm{g}}$, Angela Brennan, $\mathrm{RN}^{\mathrm{b}}$, Melanie Freeman ${ }^{\mathrm{c}, \mathrm{d}}$

${ }^{a}$ Department of Cardiology, The Royal Melbourne Hospital, Melbourne, Victoria, Australia ${ }^{b}$ Centre of Cardiovascular Research and Education in Therapeutics (CCRE), Department of Epidemiology and Preventative Medicine, Monash University, Melbourne, Victoria, Australia

${ }^{c}$ Department of Cardiology, Box Hill Hospital, Melbourne, Victoria, Australia

${ }^{d}$ Monash University Eastern Health Clinical School, Melbourne, Victoria, Australia

${ }^{e}$ Department of Cardiovascular Medicine, Alfred Hospital, Melbourne, Victoria, Australia

${ }^{f}$ Department of Cardiology, Austin Hospital, Melbourne, Victoria, Australia

${ }^{g}$ School of Public Health, Curtin University, Perth, Western Australia

On behalf of the Melbourne Interventional Group (MIG)

* Corresponding Author: Dr John Ramzy, Alfred Heart Centre, $3^{\text {rd }}$ Floor Philip Block, Alfred Hospital, 55 Commercial Rd, Melbourne 3004, AUSTRALIA. Telephone +61 423246960. E-mail Address: johnramzy@yahoo.com.au

This author takes responsibility for all aspects of the reliability and freedom from bias of the data presented and their discussed interpretation 
The Melbourne Interventional Group (MIG) gratefully acknowledges funding from Abbott, Astra-Zeneca, Medtronic, MSD, Pfizer, Servier, and The Medicines Company. These companies do not have access to data and do not have the right to review manuscripts or abstracts before publication. The MIG Research Group is supported through a National Health \& Medical Research Council of Australia Centre of Research Excellence Grant (No. 1111170) and CMR on a NHMRC Research Fellowship (No. 10458620).

Indexing Words: Coronary Artery Disease, Peripheral Arterial Disease, Revascularisation. Word Count: 4,994

Short Title for running head: Outcomes in Patients with PVD following PCI.

\section{Abstract}

Objectives: To evaluate the clinical characteristics and outcomes of patients with Peripheral Vascular Disease (PVD) undergoing Percutaneous Coronary Intervention (PCI) in a contemporary setting, and to determine whether use of drug-eluting stents (DES) improves outcomes.

Background: PVD was an independent risk factor for adverse outcomes following $\mathrm{PCl}$ in the bare-metal stent (BMS) era. It is not known whether outcomes in these patients have improved with advances in interventional techniques and stent technology, as they have for the general population. 
Methods: 18,380 patients undergoing $\mathrm{PCl}$ from an Australian registry between 2005 and 2013 were studied. Clinical and procedural data, 30-day and 12-month outcomes were compared in those with and without a reported history of PVD. Outcomes were also compared between patients with PVD who received DES and those who received BMS. Long-term mortality was compared using Australian National Death Index linkage.

Results: Patients with PVD ( $n=1,251,6.8 \%)$ were older and had more prevalent diabetes, hypertension, cerebrovascular disease, heart failure, renal impairment, ostial lesions, left main and multi-vessel disease $(p<0.001)$. Patients with PVD had significantly higher rates of major adverse cardiovascular events (MACE) compared with those without PVD, inhospital (5.7\% vs. $4.1 \%, p<0.008)$, at 30 -days (8.6\% vs. $5.8 \%, p<0.001)$ and at 12 -months (24.6\% vs. $13.2 \%, p<0.001)$. At $4.9 \pm 2.6$ years follow-up, there was significantly greater mortality in the PVD group. PVD patients who received DES experienced significantly less MACE than PVD patients treated with BMS at 30-days (4.8 vs. $10.1 \%, p<0.001)$ and $12-$ months (19.4 vs. $26.4 \%, \mathrm{p}<0.005)$.

Conclusions: PVD is an independent predictor of adverse outcomes in patients undergoing PCI. PVD patient who received DES had improved outcomes compared with those receiving BMS. 


\section{Introduction}

The incidence of lower extremity peripheral vascular disease (PVD) has grown by $23.5 \%$ in the first decade of this century. It now affects $3-12 \%$ of the world's population; around 202 million cases worldwide [1]. Patients with PVD have similar atherosclerotic risk profiles to those with coronary artery disease (CAD). In the Global Atherothrombosis Assessment (AGATHA) study, one in two patients with PVD had concomitant CAD, and one in five CAD patients had PVD [2]. Approximately 23\% of patients with both PVD and CAD have undergone percutaneous coronary intervention $(\mathrm{PCl})[3]$. Amongst the overall population of patients undergoing $\mathrm{PCl}$ advanced stent technology and more aggressive adjunctive antiplatelet and anticoagulant therapy have resulted in an improvement in outcomes $[4,5]$. However, there is evidence that this has not been the case for patients with PVD. Despite a reduction in the rate of repeat $\mathrm{PCl}$, their risk of major adverse cardiovascular events outcomes following $\mathrm{PCl}$ has remained unchanged across the early bare-metal stent (BMS) and drug-eluting stent (DES) eras [6]. While PVD is recognized as an independent predictor of poor outcomes after PCI in the BMS era [7-15], there is a paucity of data within the DES era [16]. This study aimed to evaluate whether PVD is an independent predictor of adverse outcomes outcomes in patients undergoing $\mathrm{PCl}$ in a contemporary setting. Furthermore, we aimed to investigate whether patients with PVD represent a subgroup of patients who may derive particular benefit from the use of DES over BMS. 


\section{Methods}

\subsection{Patient Population}

We analysed prospectively collected data from 18,380 consecutive patient procedures in the Melbourne Interventional Group (MIG) registry between 2005 and 2013. No procedures were excluded from the study. All procedures $(n=18,380)$ were divided into two groups. Group one consisted of procedures on patients with a history of PVD $(n=1,251)$ and group two consisted of procedures on patients without PVD $(n=17,129)$. Clinical characteristics, procedural data medication use and in-hospital, 30-day and 12-month adverse outcomes (MACE, death, recurrent myocardial infarction and target vessel revascularisation) were compared between these two groups. We further divided the PVD group into those who had received DES $(n=542)$ and those who received BMS $(n=595)$ and compared 30-day and 12-month adverse outcomes between these two subgroups. Using National Death Index (NDI) linkage, long-term mortality (mean follow-up $4.9 \pm 2.6$ years) was compared between four subgroups: patients without PVD who received DES $(n=7,653)$, patients without PVD who received BMS $(n=8,376)$, patients with PVD who received DES ( $n=539)$ and patients with PVD who received BMS ( $=594)$.

\subsection{Definitions}

Peripheral vascular disease was defined by either a history of chronic or acute occlusion or aneurysmal narrowing of the arterial lumen of the aorta or extremities and includes: claudication with exertion, extremity ischaemic rest pain, amputation for arterial insufficiency, documented aortic aneurysm, documented renal artery stenosis, positive 
non-invasive testing (e.g. ankle brachial index less than 0.9) and vascular reconstruction, bypass surgery or percutaneous intervention to the extremities [17]. Patients were not screened for asymptomatic PVD.

\subsection{Registry Design}

The Melbourne Interventional Group is a collaboration between interventional cardiologists at six major hospitals in Victoria, Australia $[18,19]$. The registry is coordinated by the Centre for Cardiovascular Research \& Education in Therapeutics, a research body within the Department of Epidemiology and Preventative Medicine Monash University, Melbourne, Australia.

The ethics committee of each participating hospital has approved the registry and all participants gave informed consent using an 'opt-out' model. A free-call number that could be used to exclude one's self from the study was provided to patients. Clinical-quality registries have recommended this model [20], which is also used by other large Australian registries [21].

Investigators at each site recorded demographic, clinical and procedural characteristics of all patients undergoing $\mathrm{PCl}$ using a case-report form with standard definitions for all fields [19]. Regular independent audits conducted on 15 verifiable variables from $5 \%$ of MIG procedures have demonstrated $96.5 \%$ accuracy [22], similar to other large registries [23].

\subsection{Procedural decisions}


Taking into account guidelines and clinical factors, the choice between balloon-only angioplasty, $\mathrm{PCl}$ with $\mathrm{BMS}$ or $\mathrm{PCl}$ with $\mathrm{DES}$ was at the discretion of the treating clinician at each centre. In Australian public (government funded) hospitals, DES are currently recommended for patients with lesions at high risk of restenosis including; diabetics, lesions greater than $18 \mathrm{~mm}$ in length, vessels less than $2.5 \mathrm{~mm}$ in diameter, in-stent restenosis and bifurcation or ostial lesions [24]. Peri-procedural antiplatelet and anticoagulant use was generally guideline based, however was also ultimately decided by each patient's treating team.

\subsection{Data Collection and Outcomes}

In-hospital complications were logged at the time of discharge from the index admission. Thirty-day and 12-month follow-up were obtained by telephone contact with the patient, their next-of-kin or treating medical practitioner, and all events were verified by case record review. Long-term mortality was determined using the Australian National Death Index (NDI) linkage, the methodology of which has been previously described in detail [25]. Essentially, The Australian NDI is a database that uses information from the births, deaths and marriages registries of each Australian state to record all deaths nation-wide. Its purpose is to enable epidemiological research. Several demographic variables (name, date of birth, age at death, date of death, gender, state/territory of registration) were used to match deceased patients in the NDI with all patients in the MIG registry. The mean \pm standard deviation NDI follow-up for patients in the MIG registry was $4.9 \pm 2.6$ years.

\subsection{Statistical Analysis}


Continuous variables are expressed as mean \pm standard deviation and categorical as numbers/percentages. The Pearson chi-squared test was used to analyse differences in discrete variables relating to characteristics, procedural data and outcomes between groups. Student's t-test or Kruskal-Wallis equality-of-populations rank test was used for continuous variables as appropriate. The Kaplan-Meier method was used to construct survival curves and the differences in survival were assessed with the log-rank test. A p value of $<0.05$ was considered to indicate statistical significance. Thirty-five baseline clinical and procedural characteristics were considered in the identification of factors independently associated with outcomes at 12-month follow-up. Factors with $p$ value $<0.1$ in simple logistic regression were considered for multiple logistic regression. Statistical analyses were carried out using Stata for Windows (Stata/MP 13.1, College Station, TX, USA). 


\section{Results}

A total of 18,380 patient procedures were enrolled in the registry between 2005 and 2013. Of these, 1,251 patients (6.8\%) had PVD and 17,129 (93.2\%) did not have a history of PVD. DES were used in $44.7 \%$ of procedures overall.

\subsection{Baseline Characteristics}

Patients with PVD were older and had a significantly greater prevalence of cardiovascular risk factors including diabetes, hypertension, dyslipidaemia and a history of $\mathrm{Ml}$ when compared to those without PVD (all p<0.001) (Table 1). They were also more likely to have a history of cerebrovascular disease, chronic pulmonary disease, heart failure, previous $\mathrm{PCl}$ and prior coronary artery bypass grafting (CABG) (all $\mathrm{p}<0.001)$. The PVD group underwent more procedures for elective and non-emergent acute coronary syndromes (ACS) while patients without PVD had a higher rate of $\mathrm{PCl}$ for ST-elevation MI (STEMI) $(p<0.001)$ (Table 1). In keeping with the lower incidence of STEMI in this group, thrombolytics and IIb/IIIa antagonists were used less often in patients with PVD $(p<0.001)$. However, despite the lower incidence of STEMI, the PVD group still had a significant number of patients presenting with out-of-hospital VF-arrest; they also had similar numbers of patients presenting with cardiogenic shock and requiring intra-aortic balloon pump use (Table 1$)$. The proportion of males was similar in both groups $(74.2 \%$ vs. $75.6 \%$, $p=0.25)$ There were more current smokers in the non-PVD group than the PVD group (24.3\% vs. $19.7 \%, p<0.001)$ (Table 1$)$. Comparison of medication use between the two groups (Tables 3 and 4) revealed a significantly greater proportion of non-PVD patients 
were taking statins at 30-days, and beta-blockers and ACE inhibitors at 30-days and 12months. Conversely, the use of ezetimibe, nitrates and calcium channel blockers was significantly higher amongst the PVD group. The PVD group were more often treated with anticoagulation in addition to single or dual-antiplatelet therapy than the non-PVD patients, who had significantly higher rates of dual-antiplatelet therapy alone.

\subsection{Angiographic characteristics}

The procedural success rate was lower in patients with PVD $(94.3 \%$ vs. 95.9\%, p<0.001). They were more likely to have multi-vessel and left main disease than those without PVD ( $76.5 \%$ vs. $58.4 \%, p<0.001$ and $3.4 \%$ vs. $0.8 \%, p<0.001$ respectively) (Table 2). There was a higher rate of in-stent restenosis amongst patients with PVD $(9.5 \%$ vs. $5.8 \%$, $\mathrm{p}<0.001)$ (Table 2). Complex lesions (ACC/AHA B2/C) and ostial lesions were more common amongst the PVD group ( $p=0.013$ and $p<0.001$ respectively), while there was no significant difference in the number of bifurcation lesions $(p=0.075)$ (Table 2$)$. Both DES and BMS use was lower in patients with PVD who had more balloon only procedures than patients without PVD $(p<0.001)$. Detailed angiographic data are shown in Table 2.

\subsection{In-Hospital, 30-Day and 12-Month Clinical Outcomes.}

Patients with PVD experienced more in-hospital MACE (5.7\% vs. 4.1\%, $p=0.008)$, driven by a significantly increased risk of death (3.8\% vs. $2.1 \%, p<0.001)$ (Table 5$)$. They also had higher rates of new renal impairment (1.9\% vs. $1.1 \%, \mathrm{p}=0.015)$, major bleeding $(3.8 \%$ vs. $2.4 \%, p=0.004)$ and peri-procedural arterial dissection at the percutaneous access site (0.6\% vs. $0.2 \%, p=0.003)$ (Table 5). 
There was a higher rate of 30 -day MACE amongst patients with PVD (8.6\% vs. 5.8\%, $\mathrm{p}<0.001)$. Death (5.0\% vs. 2.4\%, p<0.001) and new or recurrent $\mathrm{MI}(3.1 \%$ vs. $2.0 \%$, $p=0.006$ ) occurred significantly more often in the PVD group, while the rate of target vessel revascularisation (TVR) was similar in the two groups (2.2\% vs. 2.4\%, p=0.53) (Table 5).

At 12 months, patients with PVD had higher rates of MACE $(24.6 \%$ vs. $13.2 \%, p<0.001)$, death $(12.2 \%$ vs. $4.2 \%, \mathrm{p}<0.001)$, new or recurrent $\mathrm{MI}(9.8 \%$ vs. $4.4 \%, \mathrm{p}<0.001)$ and TVR (9.5\% vs. $7.2 \%, p=0.003)$ (Table 5).

Independent predictors of MACE at 12 months included PVD (OR 1.35, 95\% Cl: 1.14-1.60, $\mathrm{p}<0.001$ ), cardiogenic shock (OR 5.11, 95\% Cl: 4.14-6.30, p<0.001) and eGFR<30 $\mathrm{ml} / \mathrm{min} / 1.73 \mathrm{~m}^{2}$ (OR 3.09, 95\% Cl: 2.48-3.85, $\left.\mathrm{p}<001\right)$ (Table 6). PVD was also independently associated with 12-month mortality (OR 2.01, 95\% Cl: 1.56-2.59, p<0.001), as were cardiogenic shock and eGFR<30 $\mathrm{ml} / \mathrm{min} / 1.73 \mathrm{~m}^{2}$ (Table 7).

\subsection{PVD and DES Use}

Amongst those with PVD, 542 (43.3\%) received BMS and 595 (47.6\%) received DES with the remaining 114 (9.1\%) patients undergoing balloon angioplasty alone. There was a significantly higher prevalence of diabetes amongst the PVD patients who received DES compared with those who received BMS (54.2\% vs. 35.1\%, p<0.001) (Table 8). PVD patients receiving BMS had a higher rate of STEMI as the indication for $\mathrm{PCI}(24.4 \%$ vs. 9.4\%, p<0.001) while DES was used more often in NSTEMI, unstable angina and nonACS presentations (Table 8). When compared to the PVD patients who received DES, a higher proportion of the PVD patients receiving BMS presented with out of hospital cardiac 
arrest $(2.5 \%$ vs. $0.6 \%, p<0.008)$ and cardiogenic shock (5.9\% vs. $1.9 \%, p<0.001)$ (Table 8$)$. More patients in the PVD and DES group had complex lesions (ACC/AHA classification type B2/C) treated compared to the PVD and BMS group (59.1\% vs. 52.1\%, $\mathrm{p}=0.01)$ (Table 9). As expected, the PVD patients who received DES had higher rates of DAPT use at 30 days and 12 months (95.2\% vs. $89.3 \%, p<0.001$ and $79.8 \%$ vs. $51.4 \%, p<0.001$ respectively) (Tables 10 and 11 ).

Compared with PVD patients who received DES, patients who received BMS had a significantly higher incidence of in-hospital complications including; MACE (6.4\% vs. $2.8 \%$, $\mathrm{p}=0.004)$, death $(4.2 \%$ vs. $1.9 \%, \mathrm{p}=0.022)$ and new or recurrent $\mathrm{MI}(2.0 \%$ vs. $0.2 \%$, $\mathrm{p}=0.004$ ) (Table 12). At 30-days, patients with PVD who received DES had significantly less MACE (4.8\% vs. 10.1\%, p<0.001), death and $\mathrm{MI}$ than those who received BMS. However, the rates of TVR did not differ significantly between the two groups (1.5\% vs. 2.2\%, $p=0.96)$ (Table 12).

At 12-months, there was a significantly lower incidence of MACE $(19.4 \%$ vs. $26.4 \%$, $\mathrm{p}=0.005)$ and death $(8.5 \%$ vs. $14.6 \%, p<0.001)$ amongst patients treated with DES. However, there was no longer a difference in the rate of MI, and TVR continued to be similar (Table 12).

Over a mean follow-up period of $4.9 \pm 2.6$ years patients with PVD who received BMS had the highest mortality (38.4\%) when compared to patients with PVD who received DES (29.1\%) and those without PVD (15.3\% and 12.0\% with BMS and DES respectively, $\mathrm{p}<0.001$ ) (Figure 1). 


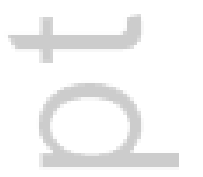

This article is protected by copyright. All rights reserved. 


\section{Discussion}

This is one of the largest registries examining outcomes in patients with PVD following PCI in the DES era. This study has shown that patients with PVD have an increased risk of short-, medium- and long-term adverse outcomes following PCI. Furthermore, PVD is an independent predictor of adverse outcomes following $\mathrm{PCl}$ in the $\mathrm{DES}$ era, despite advances in stent technology and medical therapy. Finally, an important finding of this study is that patients with PVD who were treated with DES had lower rates of death and overall major adverse cardiac events than those who received BMS.

Several studies in the BMS era concluded that PVD was an independent predictor of adverse outcomes after $\mathrm{PCl}[7-11,13-15,26,27]$. Nikolsky et al examined 12-month outcomes in 10,440 consecutive patients undergoing $\mathrm{PCl}$ in the $\mathrm{BMS}$ era, 1,969 of whom had symptomatic lower extremity PVD. The PVD group had a significantly higher 12-month mortality rate $(13.6 \%$ vs. $5.2 \%, \mathrm{p}<0.001)$, and after multivariate analysis, PVD was independently associated with increased 12-month mortality (OR 1.71, 95\% Cl 1.42-2.07, $\mathrm{p}<0.001$ ) [15]. Similarly, Singh et al found that, even after adjustment for concomitant risk factors, patients with PVD treated with $\mathrm{PCl}$ in the $\mathrm{BMS}$ era had an $84 \%$ relative-risk increase in in-hospital mortality and a $48 \%$ relative-risk increase in death at 3 year followup compared to patients without PVD [7]. Analysis of the Tirofiban and Reopro Give Similar Efficacy Outcome Trial (TARGET) showed that PVD was independently associated with a 2 to 3 fold increase in mortality 12 months after $\mathrm{PCl}$ [10]. Amongst patients undergoing $\mathrm{PCl}$ for MI, PVD was independently associated with a doubling of in-hospital 
mortality, and the number of diseased vascular beds in patients with PVD was associated with a graded increase in the risk of adverse outcomes [26].

Similar to findings in the BMS era, our study suggests that PVD continues to be independently associated with roughly a two-fold increased risk of 12-month mortality post$\mathrm{PCl}$ in the DES era.

This study also examined differences in outcomes between patients with PVD who received DES and those PVD patients receiving BMS. Interestingly, we found that patients with PVD who received DES had reduced short-, medium- and long-term MACE and mortality despite no difference in TVR. The difference in mortality was seen out to mean follow-up of $4.9 \pm 2.6$ years. In stent-restenosis and the need for TVR are greatest in the first year after stent insertion and this is when DES have been shown to be superior to BMS in reducing the need for TVR, but not mortality [28]. In the absence of a reduction in TVR, there is no clear explanation for the reduced short- and long-term MACE and mortality in our PVD patients who received DES compared to those who received BMS. However, in this cohort we found a significantly higher rate of $\mathrm{PCl}$ performed for in-stent restenosis in the PVD group. Our data suggests that outcomes for patients with PVD undergoing real world $\mathrm{PCl}$ could possibly be improved by greater use of drug-eluting stents over bare-metal stents in this particular group of patients.

Prior to our registry, only two other studies have compared outcomes between patients with PVD and those without PVD in the DES era $[16,29]$. Midwall et al investigated outcomes amongst 173 patients with PVD and 2,282 patients without a history of PVD who 
underwent $\mathrm{PCl}$ in the early-DES era [16]. The PVD group had significantly higher unadjusted rates of death than the non-PVD group in-hospital $(1.8 \%$ vs. $0.1 \%, p=0.006)$, at 12-months (8.2 vs. 2.9, $\mathrm{p}=0.001)$ and 4 -years (23.8\% vs. $10.8 \%, \mathrm{p}<0.001)$. However, after adjustment for differences in the patient's clinical and angiographic characteristics, PVD was no longer an independent predictor of mortality [16]. In contrast, our study found that PVD remained an independent predictor of adverse outcomes after multivariate adjustment. Of note, the study by Midwall et al had a smaller number of patients and may have been underpowered to detect a true mortality difference after multivariate adjustment. Additionally, the definition of PAD in Midwall's study allowed for the inclusion of patients without PAD of the aorta, renal arteries or extremities, but who had only carotid artery disease or a history of cerebrovascular accident. These patients were not included in our PVD cohort, all of whom had PVD involving the aorta, renal arteries or extremeties. There is some evidence that cerebrovascular disease may be a lesser risk factor for adverse outcomes after $\mathrm{PCl}$ than peripheral arterial disease $[11,14]$ hence their inclusion in the PVD cohort may have reduced the apparent overall increased risk amongst their PVD group.

More recently, an analysis of the Assessment of Dual AntiPlatelet Therapy with Drug Eluting Stents (ADAPT-DES) study was conducted to determine the relationship between PVD, platelet reactivity and subsequent adverse outcomes. There was a $10.2 \%$ prevalence of PVD amongst the 8,582 patients included in the analysis, all of whom received drug-eluting stents. In-line with our findings, PAD was found to be an independent predictor of MACE (adjusted HR 1.34, $\mathrm{p}=0.003$ ), mortality, MI and bleeding at 
two year follow-up. The increased risk amongst PVD patients was not mediated by heightened platelet reactivity (HPR), which affected both PVD and non-PVD patients similarly [29].

The increased rate of adverse outcomes observed in our PVD group may be related to their higher rate of periprocedural bleeding events (3.8\% vs. $2.4 \%, p=0.004)$. Amongst the general population of patients undergoing $\mathrm{PCl}$, periprocedural bleeding has been associated with a 3 to 10-fold increased risk of in-hospital and 30-day mortality [30-34] and a 2 to 4.5-fold increased risk of 12-month mortality [30, 34-37]. Bleeding has also been associated with a greater risk of myocardial infarction and stroke [30, 34]. Analysis of the Harmonizing Outcomes with Revascularization and Stents in Acute Myocardial Infarction (HORIZONS-AMI) trial found an increased risk of MACE and mortality that persisted out to 3 years post-PCI in STEMI patients who had an in-hospital major bleed [38]. Patients with vascular disease may be less able to control the systemic amplification of local clotting mechanisms triggered by bleeding because the counter regulatory pathways are primarily active within endothelial cells. This may predispose them to a hypercoagulable state following a bleeding event [39]. In addition, periprocedural bleeding may lead to antiplatelet or antithrombotic therapy being withheld, which may contribute to the risk of thrombotic complications. Of the patients who experienced bleeding in the Global Registry of Acute Coronary Events (GRACE) registry, there was higher in-hospital mortality in those who had their aspirin, thienopyridine or low-molecular-weight heparin ceased, compared with those in whom the medications were continued in spite of bleeding [40]. Furthermore, 
blood transfusion after $\mathrm{PCl}$ may itself independently increase risk of 12-month mortality $(R R=2.42, p=0.0045)$ amongst patients with severe bleeding [41].

In addition to carrying their own mortality risks, greater age and a higher proportion of comorbidities may influence clinicians to treat residual CAD in these patients less aggressively. Results from the 'Get With the Guidelines' Program that included 37,633 ACS patients with a history of vascular disease found that these patients had a higher adjusted in-hospital mortality than those without a history of vascular disease, yet received less statins, ACE inhibitors and smoking cessation advice [42]. Our analysis suggests that patients with PVD are less often treated with medications that carry prognostic benefit (beta-blockers and ACE-inhibitors) and more often treated with those that portend a symptomatic benefit (nitrates and calcium channel blockers). The increased age and comorbidities among patients with PVD may also negatively impact their participation in exercise-based cardiac rehabilitation, which has been shown to improve medium and longterm mortality in patients after coronary revascularisation [43].

This study highlights the importance of identifying patients with PVD undergoing PCI. Asymptomatic PVD, as detected by non-invasive testing, is 3 to 4 times more common than symptomatic PVD [15]. In a substudy of the Bypass Angioplasty Revascularization (BARI) trial, asymptomatic PVD yielded the same increased mortality risk as symptomatic PVD amongst patients who underwent coronary revascularisation [9]. Seventy per cent of the patients with lower extremity arterial disease in that study would not have been identified without the use of ABI. Reflecting these findings, the 2005 ACCF/AHA guideline for the management of patients with peripheral artery disease was updated in 2011 to 
include a class one recommendation for ankle-brachial index screening for all patients over 65 regardless of symptoms or risk factors [44]. The detection of PVD is important, as it can be a marker of other underlying comorbidities such as CAD and diabetes mellitus that may require intervention.

There were some limitations to this analysis. A registry-based observational study has inherent limitations with data collected prospectively but analysed retrospectively. The reliance on symptomatic reporting and historical evidence in identifying patients with PVD likely resulted in under-recognition of patients with the condition. Finally, patients with severe PVD may have been more likely to be treated medically, thus skewing the population of PVD patients included in our interventional registry.

\section{Conclusion}

PVD is an independent predictor of adverse outcomes following $\mathrm{PCl}$ in the contemporary setting. Further studies are warranted to elucidate the mechanisms responsible for this. The improvement in long-term outcomes seen with drug eluting-stent use was significantly greater for PVD patients than non-PVD patients, suggesting that operators should particularly preference the use of DES over BMS for this high-risk cohort.

\section{References}

[1] Fowkes FG, Rudan D, Rudan I, Aboyans V, Denenberg JO, McDermott MM, et al. Comparison of global estimates of prevalence and risk factors for peripheral artery disease in 2000 and 2010: a systematic review and analysis. Lancet. 2013;382:1329-40. 
[2] Fowkes FG, Low LP, Tuta S, Kozak J, Investigators A. Ankle-brachial index and extent of atherothrombosis in 8891 patients with or at risk of vascular disease: results of the international AGATHA study. European heart journal. 2006;27:1861-7.

[3] Hirsch AT, Criqui MH, Treat-Jacobson D, Regensteiner JG, Creager MA, Olin JW, et al. Peripheral arterial disease detection, awareness, and treatment in primary care. Jama. 2001;286:1317-24.

[4] Singh M, Rihal CS, Berger PB, Bell MR, Grill DE, Garratt KN, et al. Improving outcome over time of percutaneous coronary interventions in unstable angina. Journal of the American College of Cardiology. 2000;36:674-8.

[5] Williams DO, Holubkov R, Yeh W, Bourassa MG, Al-Bassam M, Block PC, et al. Percutaneous coronary intervention in the current era compared with 1985-1986: the National Heart, Lung, and Blood Institute Registries. Circulation. 2000;102:2945-51.

[6] Parikh SV, Saya S, Divanji P, Banerjee S, Selzer F, Abbott JD, et al. Risk of death and myocardial infarction in patients with peripheral arterial disease undergoing percutaneous coronary intervention (from the National Heart, Lung and Blood Institute Dynamic Registry). The American journal of cardiology. 2011;107:959-64.

[7] Singh M, Lennon RJ, Darbar D, Gersh BJ, Holmes DR, Jr., Rihal CS. Effect of peripheral arterial disease in patients undergoing percutaneous coronary intervention with intracoronary stents. Mayo Clinic proceedings. 2004;79:1113-8.

[8] Berger JS, Petersen JL, Brown DL. Vascular disease burden and in-hospital outcomes among patients undergoing percutaneous coronary intervention in New York State. Circulation Cardiovascular interventions. 2009;2:317-22.

[9] Burek KA, Sutton-Tyrrell K, Brooks MM, Naydeck B, Keller N, Sellers MA, et al. Prognostic importance of lower extremity arterial disease in patients undergoing coronary revascularization in the Bypass Angioplasty Revascularization Investigation (BARI). Journal of the American College of Cardiology. 1999;34:716-21.

[10] Chiu JH, Topol EJ, Whitlow PL, Hsu AP, Tuzcu EM, Franco I, et al. Peripheral vascular disease and one-year mortality following percutaneous coronary revascularization. The American journal of cardiology. 2003;92:582-3.

[11] Morikami Y, Natsuaki M, Morimoto T, Ono K, Nakagawa Y, Furukawa Y, et al. Impact of polyvascular disease on clinical outcomes in patients undergoing coronary revascularization: an observation from the CREDO-Kyoto Registry Cohort-2. Atherosclerosis. 2013;228:426-31. [12] Nikolsky E, Mehran R, Dangas GD, Lasic Z, Mintz GS, Negoita M, et al. Prognostic significance of cerebrovascular and peripheral arterial disease in patients having percutaneous coronary interventions. American Journal of Cardiology.93:1536-9. [13] Saw J, Bhatt DL, Moliterno DJ, Brener SJ, Steinhubl SR, Lincoff AM, et al. The influence of peripheral arterial disease on outcomes: a pooled analysis of mortality in eight large randomized percutaneous coronary intervention trials. Journal of the American College of Cardiology. 2006;48:1567-72.

[14] Nikolsky E, Mehran R, Dangas GD, Lasic Z, Mintz GS, Negoita M, et al. Prognostic significance of cerebrovascular and peripheral arterial disease in patients having percutaneous coronary interventions. The American journal of cardiology. 2004;93:1536-9. 
[15] Nikolsky E, Mehran R, Mintz GS, Dangas GD, Lansky AJ, Aymong ED, et al. Impact of symptomatic peripheral arterial disease on 1-year mortality in patients undergoing percutaneous coronary interventions. Journal of endovascular therapy : an official journal of the International Society of Endovascular Specialists. 2004;11:60-70.

[16] Midwall S, Swaminathan RV, Charitakis K, Kim LK, Gordin J, Hriljac I, et al. Impact of peripheral vascular disease on short- and long-term outcomes in patients undergoing nonemergent percutaneous coronary intervention in the drug-eluting stent era. The Journal of invasive cardiology. 2013;25:132-6.

[17] Cannon CP, Battler A, Brindis RG, Cox JL, Ellis SG, Every NR, et al. American College of Cardiology key data elements and definitions for measuring the clinical management and outcomes of patients with acute coronary syndromes. A report of the American College of Cardiology Task Force on Clinical Data Standards (Acute Coronary Syndromes Writing Committee). Journal of the American College of Cardiology. 2001;38:2114-30.

[18] Ajani AE, Szto G, Duffy SJ, Eccleston D, Clark DJ, Lefkovits J, et al. The foundation and launch of the Melbourne Interventional Group: a collaborative interventional cardiology project. Heart, lung \& circulation. 2006;15:44-7.

[19] Chan W, Clark DJ, Ajani AE, Yap CH, Andrianopoulos N, Brennan AL, et al. Progress towards a National Cardiac Procedure Database--development of the Australasian Society of Cardiac and Thoracic Surgeons (ASCTS) and Melbourne Interventional Group (MIG) registries. Heart, lung \& circulation. 2011;20:10-8.

[20] McNeil JJ, Evans SM, Johnson NP, Cameron PA. Clinical-quality registries: their role in quality improvement. The Medical journal of Australia. 2010;192:244-5.

[21] Graves SE, Davidson D, Ingerson L, Ryan P, Griffith EC, McDermott BF, et al. The Australian Orthopaedic Association National Joint Replacement Registry. The Medical journal of Australia. 2004;180:S31-4.

[22] Andrianopoulos N, Dinh D, Duffy SJ, Clark DJ, Brennan AL, Chan W, et al. Quality control activities associated with registries in interventional cardiology and surgery. Heart, lung \& circulation. 2011;20:180-6.

[23] Lagerqvist B, James SK, Stenestrand U, Lindback J, Nilsson T, Wallentin L, et al. Long-term outcomes with drug-eluting stents versus bare-metal stents in Sweden. The New England journal of medicine. 2007;356:1009-19.

[24] Yan BP, Ajani AE, Duffy SJ, New G, Horrigan M, Szto G, et al. Use of drug-eluting stents in Victorian public hospitals. The Medical journal of Australia. 2006;185:363-7.

[25] Wilson WM, Andrianopoulos N, Clark D, Duffy SJ, Brennan A, Harries I, et al. Long-term predictors of mortality after percutaneous coronary intervention in the era of drug-eluting stents. The American journal of cardiology. 2011;108:936-42.

[26] Jeremias A, Gruberg L, Patel J, Connors G, Brown DL. Effect of peripheral arterial disease on in-hospital outcomes after primary percutaneous coronary intervention for acute myocardial infarction. The American journal of cardiology. 2010;105:1268-71.

[27] Guerrero M, Harjai K, Stone GW, Brodie B, Cox D, Boura J, et al. Usefulness of the presence of peripheral vascular disease in predicting mortality in acute myocardial infarction patients treated with primary angioplasty (from the Primary Angioplasty in Myocardial Infarction Database). The American journal of cardiology. 2005;96:649-54. 
[28] Weisz G, Leon MB, Holmes DR, Jr., Kereiakes DJ, Popma JJ, Teirstein PS, et al. Five-year follow-up after sirolimus-eluting stent implantation results of the SIRIUS (Sirolimus-Eluting Stent in De-Novo Native Coronary Lesions) Trial. Journal of the American College of Cardiology. 2009;53:1488-97.

[29] Stone GW, Witzenbichler B, Weisz G, Rinaldi MJ, Neumann FJ, Metzger DC, et al. Platelet reactivity and clinical outcomes after coronary artery implantation of drug-eluting stents (ADAPT-DES): a prospective multicentre registry study. Lancet. 2013;382:614-23. [30] Yatskar L, Selzer F, Feit F, Cohen HA, Jacobs AK, Williams DO, et al. Access site hematoma requiring blood transfusion predicts mortality in patients undergoing percutaneous coronary intervention: data from the National Heart, Lung, and Blood Institute Dynamic Registry. Catheterization and cardiovascular interventions : official journal of the Society for Cardiac Angiography \& Interventions. 2007;69:961-6.

[31] Chhatriwalla AK, Amin AP, Kennedy KF, House JA, Cohen DJ, Rao SV, et al. Association between bleeding events and in-hospital mortality after percutaneous coronary intervention. Jama. 2013;309:1022-9.

[32] Hochholzer W, Wiviott SD, Antman EM, Contant CF, Guo J, Giugliano RP, et al. Predictors of bleeding and time dependence of association of bleeding with mortality: insights from the Trial to Assess Improvement in Therapeutic Outcomes by Optimizing Platelet Inhibition With Prasugrel--Thrombolysis in Myocardial Infarction 38 (TRITON-TIMI 38). Circulation. 2011;123:2681-9.

[33] Kinnaird TD, Stabile E, Mintz GS, Lee CW, Canos DA, Gevorkian N, et al. Incidence, predictors, and prognostic implications of bleeding and blood transfusion following percutaneous coronary interventions. The American journal of cardiology. 2003;92:930-5. [34] Rao SV, Dai D, Subherwal S, Weintraub WS, Brindis RS, Messenger JC, et al. Association between periprocedural bleeding and long-term outcomes following percutaneous coronary intervention in older patients. JACC Cardiovascular interventions. 2012;5:958-65.

[35] Feit F, Voeltz MD, Attubato MJ, Lincoff AM, Chew DP, Bittl JA, et al. Predictors and impact of major hemorrhage on mortality following percutaneous coronary intervention from the REPLACE-2 Trial. The American journal of cardiology. 2007;100:1364-9.

[36] Ndrepepa G, Berger PB, Mehilli J, Seyfarth M, Neumann FJ, Schomig A, et al.

Periprocedural bleeding and 1-year outcome after percutaneous coronary interventions: appropriateness of including bleeding as a component of a quadruple end point. Journal of the American College of Cardiology. 2008;51:690-7.

[37] Wlodarczyk J, Ajani AE, Kemp D, Andrianopoulos N, Brennan AL, Duffy SJ, et al. Incidence, Predictors and Outcomes of Major Bleeding in Patients Following Percutaneous Coronary Interventions in Australia. Heart, lung \& circulation. 2016;25:107-17.

[38] Suh JW, Mehran R, Claessen BE, Xu K, Baber U, Dangas G, et al. Impact of in-hospital major bleeding on late clinical outcomes after primary percutaneous coronary intervention in acute myocardial infarction the HORIZONS-AMI (Harmonizing Outcomes With Revascularization and Stents in Acute Myocardial Infarction) trial. Journal of the American College of Cardiology. 2011;58:1750-6.

[39] Lane DA, Philippou H, Huntington JA. Directing thrombin. Blood. 2005;106:2605-12. 
[40] Spencer FA, Moscucci M, Granger CB, Gore JM, Goldberg RJ, Steg PG, et al. Does comorbidity account for the excess mortality in patients with major bleeding in acute myocardial infarction? Circulation. 2007;116:2793-801.

[41] Kim P, Dixon S, Eisenbrey AB, O'Malley B, Boura J, O'Neill W. Impact of acute blood loss anemia and red blood cell transfusion on mortality after percutaneous coronary intervention. Clinical cardiology. 2007;30:II35-43.

[42] Brilakis ES, Hernandez AF, Dai D, Peterson ED, Banerjee S, Fonarow GC, et al. Quality of care for acute coronary syndrome patients with known atherosclerotic disease: results from the Get With the Guidelines Program. Circulation. 2009;120:560-7.

[43] Heran BS, Chen JM, Ebrahim S, Moxham T, Oldridge N, Rees K, et al. Exercise-based cardiac rehabilitation for coronary heart disease. The Cochrane database of systematic reviews. 2011:CD001800.

[44] Rooke TW, Hirsch AT, Misra S, Sidawy AN, Beckman JA, Findeiss LK, et al. 2011 ACCF/AHA Focused Update of the Guideline for the Management of Patients With Peripheral Artery Disease (updating the 2005 guideline): a report of the American College of Cardiology Foundation/American Heart Association Task Force on Practice Guidelines. Journal of the American College of Cardiology. 2011;58:2020-45.

\section{Figure Legend}

\section{Figure 1}

Long-Term Kaplan-Meier Survival in patients; without Peripheral Vascular Disease (PVD) receiving Drug-eluting Stents (DES), without PVD receiving Bare-Metal Stents (BMS), with PVD receiving DES, and with PVD receiving BMS. 


\begin{tabular}{|c|c|c|c|}
\hline & $\begin{array}{c}\text { PVD } \\
(\mathrm{N}=1,251)\end{array}$ & $\begin{array}{c}\text { No PVD } \\
(\mathrm{N}=17,129)\end{array}$ & $\begin{array}{c}p \\
\text { value }\end{array}$ \\
\hline Mean age - years & $71.0 \pm 10$ & $64 \pm 12$ & $<0.001$ \\
\hline Male gender & $928(74.2 \%)$ & $12,956(75.6 \%)$ & 0.25 \\
\hline \multicolumn{4}{|l|}{ Medical history } \\
\hline Diabetes mellitus & $564(45.1 \%)$ & $3,998(23.4 \%)$ & $<0.001$ \\
\hline Smoking (current) & $244(19.7 \%)$ & $4,137(24.3 \%)$ & $<0.001$ \\
\hline Chronic lung disease & $219(17.6 \%)$ & $1,627(9.5 \%)$ & $<0.001$ \\
\hline Hypertension & $1,088(87.0 \%)$ & $11,206(65.4 \%)$ & $<0.001$ \\
\hline Dyslipidaemia & $1,076(86.1 \%)$ & $11,905(69.6 \%)$ & $<0.001$ \\
\hline Family history of CAD & $464(37.9 \%)$ & 6,596 (38.9\%) & 0.524 \\
\hline Cerebrovascular disease & $282(22.6 \%)$ & $943(5.5 \%)$ & $<0.001$ \\
\hline Heart failure & $154(12.32 \%)$ & $557(3.3 \%)$ & $<0.001$ \\
\hline Previous MI & $608(48.6 \%)$ & $4,495(26.3 \%)$ & $<0.001$ \\
\hline Previous PCl & $441(35.3 \%)$ & $4,221(24.65 \%)$ & $<0.001$ \\
\hline Previous CABG & $311(24.9 \%)$ & $1,308(7.6 \%)$ & $<0.001$ \\
\hline eGFR & & & $<0.001$ \\
\hline$<30$ & $163(13.2 \%)$ & $400(2.4 \%)$ & \\
\hline $30-59$ & $397(32.2 \%)$ & $3,273(19.6 \%)$ & \\
\hline$>60$ & $675(54.7 \%)$ & $13,065(78.1 \%)$ & \\
\hline
\end{tabular}




\begin{tabular}{|c|c|c|c|}
\hline Mean Baseline Serum $\mathrm{Cr}$ & $140.8 \pm 144.7$ & $93.2 \pm 66.3$ & $<0.001$ \\
\hline Mean BMI $\left(\mathrm{kg} / \mathrm{m}^{2}\right)$ & $27.8(27.4-28.1)$ & $28.4(28.3-28.5)$ & $<0.001$ \\
\hline \multicolumn{4}{|l|}{ LVEF } \\
\hline$<30 \%$ & $53(5.0 \%)$ & $350(2.35 \%)$ & \multirow{3}{*}{$<0.001$} \\
\hline $30-45 \%$ & $325(30.8 \%)$ & $3,384(22.7 \%)$ & \\
\hline$>45 \%$ & $677(64.2 \%)$ & $11,172(75.0 \%)$ & \\
\hline \multicolumn{4}{|l|}{ Indication for index $\mathrm{PCl}$} \\
\hline STEMI & $217(17.4 \%)$ & $5,249(30.7 \%)$ & \multirow{4}{*}{$<0.001$} \\
\hline NSTEMI & $413(33.0 \%)$ & $4,503(26.3 \%)$ & \\
\hline Unstable angina & $127(10.2 \%)$ & $1,669(9.8 \%)$ & \\
\hline Non-ACS & $493(39.4 \%)$ & $5,696(33.3 \%)$ & \\
\hline Out of hospital cardiac arrest & $21(1.7 \%)$ & $437(2.6 \%)$ & 0.056 \\
\hline Cardiogenic shock & $52(4.2 \%)$ & $550(3.2 \%)$ & 0.070 \\
\hline IABP & $27(2.2 \%)$ & $422(2.5 \%)$ & 0.50 \\
\hline \multicolumn{4}{|l|}{ Medications } \\
\hline Thrombolytics & $22(1.8 \%)$ & $801(4.7 \%)$ & $<0.001$ \\
\hline IIb/IIla Blockade & $263(21.1 \%)$ & $5,399(31.5 \%)$ & $<0.001$ \\
\hline Heparin & $1229(98.2 \%)$ & $16726(97.7 \%)$ & 0.21 \\
\hline LMWH & $239(19.1 \%)$ & $3272(19.1 \%)$ & 0.99 \\
\hline Bivalirudin & $13(1.1 \%)$ & $258(1.6 \%)$ & 0.15 \\
\hline Aspirin & $1239(99.0 \%)$ & $16900(98.7 \%)$ & 0.28 \\
\hline Clopidogrel & 1167 (93.4\%) & $15918(93.0 \%)$ & 0.55 \\
\hline
\end{tabular}

This article is protected by copyright. All rights reserved. 


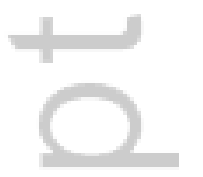

This article is protected by copyright. All rights reserved. 


\begin{tabular}{|c|c|c|c|}
\hline & $\begin{array}{c}\text { PVD } \\
(N=1,251)\end{array}$ & $\begin{array}{c}\text { No PVD } \\
(\mathrm{N}=17,129)\end{array}$ & $\begin{array}{c}\text { p } \\
\text { value }\end{array}$ \\
\hline \multicolumn{4}{|l|}{ Procedure status } \\
\hline Elective & $502(40.3 \%)$ & $5,866(34.3 \%)$ & $<0.001$ \\
\hline Urgent & $739(59.1 \%)$ & $10,985(64.1 \%)$ & \\
\hline Rescue & $10(0.8 \%)$ & $278(1.6 \%)$ & \\
\hline Percutaneous entry location & & & $<0.001$ \\
\hline Femoral & $1,079(86.3 \%)$ & $15,412(90.0 \%)$ & \\
\hline Radial & $137(11.0 \%)$ & $1,671(9.8 \%)$ & \\
\hline Disease extent & & & $<0.001$ \\
\hline Single-vessel disease & $293(23.5 \%)$ & $7,086(41.6 \%)$ & \\
\hline Multi-vessel disease & $954(76.5 \%)$ & $9,963(58.4 \%)$ & \\
\hline \multicolumn{4}{|l|}{ Culprit vessel } \\
\hline Left main coronary artery & $51(3.4 \%)$ & $170(0.8 \%)$ & $<0.001$ \\
\hline Proximal LAD & $193(12.7 \%)$ & $3,302(16.3 \%)$ & $<0.001$ \\
\hline LAD & $415(27.4 \%)$ & $7,057(34.8 \%)$ & $<0.001$ \\
\hline LCX & $228(15.1 \%)$ & $2,668(13.2 \%)$ & 0.036 \\
\hline $\mathrm{RCA}$ & $480(31.7 \%)$ & $6,496(32.1 \%)$ & 0.77 \\
\hline Grafts & $145(9.6 \%)$ & $491(2.4 \%)$ & $<0.001$ \\
\hline Type of coronary lesion & & & $<0.001$ \\
\hline
\end{tabular}




\begin{tabular}{|c|c|c|c|}
\hline De novo & $1,362(89.9 \%)$ & $19,034(93.9 \%)$ & \\
\hline Restenosis (no prior stent) & $9(0.6 \%)$ & $66(0.3 \%)$ & \\
\hline In stent restenosis & $144(9.5 \%)$ & 1,165 (5.8\%) & \\
\hline $\begin{array}{l}\text { Mean no of } \quad \text { lesions } \\
\text { treated/patient }\end{array}$ & $1.2 \pm 0.5$ & $1.2 \pm 0.4$ & 0.02 \\
\hline $\begin{array}{l}\text { Mean no. of stents } \\
\text { deployed/procedure }\end{array}$ & $1.2 \pm 0.7$ & $1.2 \pm 0.6$ & 0.90 \\
\hline Lesion type & & & \\
\hline $\mathrm{B} 2$ and $\mathrm{C}$ & $869(57.4 \%)$ & $10,960(54.1 \%)$ & 0.013 \\
\hline Mean stent diameter (mm\%) & $2.9 \pm 0.5$ & $2.9 \pm 0.5$ & 0.20 \\
\hline Mean stent length (mm\%) & $16.9 \pm 5.3$ & $17.3 \pm 5.6$ & 0.009 \\
\hline Bifurcation lesion & $142(9.4 \%)$ & $2,197(10.8 \%)$ & 0.075 \\
\hline Ostial lesion & $142(9.4 \%)$ & $1,368(6.8 \%)$ & $<0.001$ \\
\hline \multicolumn{4}{|l|}{ Type of stent } \\
\hline Balloon only & $114(9.1 \%)$ & $1,066(6.2 \%)$ & $<0.001$ \\
\hline Bare metal & $595(47.6 \%)$ & $8,386(49.0 \%)$ & \\
\hline Drug eluting & $542(43.3 \%)$ & $7,677(44.8 \%)$ & \\
\hline Procedural success rate & $1357(94.3 \%)$ & $17,846(95.9 \%)$ & 0.004 \\
\hline
\end{tabular}




\begin{tabular}{|c|c|c|c|}
\hline & $\begin{array}{c}\text { PVD } \\
(N=1,090)\end{array}$ & $\begin{array}{c}\text { No PVD } \\
(\mathrm{N}=15,601)\end{array}$ & $\begin{array}{c}p \\
\text { value }\end{array}$ \\
\hline \multicolumn{4}{|l|}{ Antiplatelet/Anticoagulant } \\
\hline No AP and no AC & $4(0.4 \%)$ & $34(0.2 \%)$ & 0.318 \\
\hline Aspirin only & $52(4.8 \%)$ & $593(3.8 \%)$ & 0.108 \\
\hline Thienopyridine only & $22(2.0 \%)$ & $193(1.2 \%)$ & 0.027 \\
\hline Aspirin and Thienopyridine & $985(89.6 \%)$ & $14,673(93.2 \%)$ & $<0.001$ \\
\hline Anticoagulation only & $6(0.6 \%)$ & $19(0.1 \%)$ & $<0.001$ \\
\hline Single AP and AC & $30(2.8 \%)$ & $213(1.4 \%)$ & $<0.001$ \\
\hline Triple therapy & $78(7.2 \%)$ & $813(5.2 \%)$ & 0.006 \\
\hline Statin & $998(91.6 \%)$ & $14,816(94.7)$ & $<0.001$ \\
\hline Fibrate & $24(2.6 \%)$ & $224(1.8 \%)$ & 0.070 \\
\hline Ezetimibe & $100(10.8 \%)$ & $586(4.6 \%)$ & $<0.001$ \\
\hline Beta Blocker & $774(71.3 \%)$ & $12,142(77.9 \%)$ & $<0.001$ \\
\hline ACE inhibitor & $599(55.3 \%)$ & $9,942(63.8 \%)$ & $<0.001$ \\
\hline ARB & $287(26.5 \%)$ & $2,722(17.5 \%)$ & $<0.001$ \\
\hline Nitrate & $200(21.8 \%)$ & 1, $056(8.4 \%)$ & $<0.001$ \\
\hline Ca Channel Blocker & $268(29.1 \%)$ & 1, 975 (15.7) & $<0.001$ \\
\hline
\end{tabular}




\begin{tabular}{|c|c|c|c|}
\hline & $\begin{array}{c}\text { PVD } \\
(\mathrm{N}=966)\end{array}$ & $\begin{array}{c}\text { No PVD } \\
(\mathrm{N}=14,696)\end{array}$ & $\begin{array}{c}p \\
\text { value }\end{array}$ \\
\hline \multicolumn{4}{|l|}{ Antiplatelet/Anticoagulant } \\
\hline No AP and no AC & $13(1.4 \%)$ & $167(1.1 \%)$ & 0.528 \\
\hline Aspirin only & $231(23.9 \%)$ & $3,890(26.1 \%)$ & 0.121 \\
\hline Thienopyridine only & $39(4.0 \%)$ & $493(3.3 \%)$ & 0.230 \\
\hline Aspirin and Thienopyridine & $619(63.7 \%)$ & $9,785(65.6 \%)$ & 0.233 \\
\hline Anticoagulation only & $14(1.5 \%)$ & $47(0.3 \%)$ & 0.000 \\
\hline Single $A P$ and $A C$ & $52(5.4 \%)$ & $520(3.5 \%)$ & 0.002 \\
\hline Triple therapy & $34(3.5 \%)$ & $377(2.5 \%)$ & 0.006 \\
\hline Statin & $882(91.2 \%)$ & $13,828(92.7)$ & 0.079 \\
\hline Fibrate & $25(2.7 \%)$ & $291(2.1 \%)$ & 0.211 \\
\hline Ezetimibe & $114(12.4 \%)$ & $943(6.8 \%)$ & $<0.001$ \\
\hline Beta Blocker & $630(65.8 \%)$ & 10, $564(71.3 \%)$ & $<0.001$ \\
\hline ACE inhibitor & $490(51.3 \%)$ & $8,718(58.9 \%)$ & $<0.001$ \\
\hline ARB & $276(28.8 \%)$ & $3,154(21.3 \%)$ & $<0.001$ \\
\hline Nitrate & $153(16.7 \%)$ & $1,160(8.4 \%)$ & $<0.001$ \\
\hline Ca Channel Blocker & $302(33.2 \%)$ & 2, 406 (17.5) & $<0.001$ \\
\hline
\end{tabular}


Table 5. In-Hospital, 30-Day and 12-Month Clinical Outcomes

\begin{tabular}{|c|c|c|c|}
\hline & $\begin{array}{c}\text { PVD } \\
(N=1,251)\end{array}$ & $\begin{array}{c}\text { No PVD } \\
(\mathrm{N}=17,129)\end{array}$ & $p$ value \\
\hline \multicolumn{4}{|l|}{ In-Hospital } \\
\hline MACE & $71(5.7 \%)$ & $705(4.1 \%)$ & 0.008 \\
\hline Death & $47(3.8 \%)$ & $357(2.1 \%)$ & $<0.001$ \\
\hline New or recurrent $\mathrm{MI}$ & $17(1.4 \%)$ & $199(1.2 \%)$ & 0.534 \\
\hline Repeat PCl & $3(0.2 \%)$ & $108(0.6 \%)$ & 0.084 \\
\hline CABGs & $10(0.8 \%)$ & $148(0.9 \%)$ & 0.806 \\
\hline New Renal Impairment & $24(1.9 \%)$ & $196(1.1 \%)$ & 0.015 \\
\hline Bleeding event & $47(3.8 \%)$ & $418(2.4 \%)$ & 0.004 \\
\hline Site of bleeding & & & 0.698 \\
\hline Retroperitoneal & $4(8.7 \%)$ & $24(6 \%)$ & \\
\hline Percutaneous entry site & $16(34.8 \%)$ & $157(39.4 \%)$ & \\
\hline Other & $26(56.5 \%)$ & $218(54.6 \%)$ & \\
\hline \multicolumn{4}{|l|}{ Vascular Complication } \\
\hline Access Site Occlusion & 0 & $6(0.04 \%)$ & 0.501 \\
\hline Loss of distal pulse & $1(0.1 \%)$ & $17(0.1 \%)$ & 0.833 \\
\hline Peripheral Arterial Dissection & $7(0.6 \%)$ & $29(0.2 \%)$ & 0.003 \\
\hline AV Fistula & $1(0.1 \%)$ & $9(0.1 \%)$ & 0.688 \\
\hline Pseudoaneurysm & $1(0.1 \%)$ & $56(0.3 \%)$ & 0.129 \\
\hline
\end{tabular}




\begin{tabular}{lccc}
\hline 30-Days & & & \\
MACE & $107(8.6 \%)$ & $996(5.8 \%)$ & $<0.001$ \\
Death & $62(5.0 \%)$ & $416(2.4 \%)$ & $<0.001$ \\
\multicolumn{1}{c}{ Cardiac cause } & $48(77.4 \%)$ & $329(79.3 \%)$ & 0.737 \\
Myocardial infarction & $39(3.12 \%)$ & $339(2.0 \%)$ & 0.006 \\
TVR & $27(2.2 \%)$ & $418(2.4 \%)$ & 0.531 \\
\hline 12-Months & $308(24.6 \%)$ & $2264(13.2 \%)$ & $<0.001$ \\
MACE & $153(12.2 \%)$ & $720(4.2 \%)$ & $<0.001$ \\
Death & $90(58.8 \%)$ & $431(60.1 \%)$ & 0.768 \\
\multicolumn{1}{c}{ Cardiac cause } & $123(9.8 \%)$ & $758(4.4 \%)$ & $<0.001$ \\
Myocardial infarction & $119(9.5 \%)$ & $1236(7.2 \%)$ & 0.003 \\
TVR & & & \\
\hline
\end{tabular}


Table 6. Predictors of 12-Month MACE

\begin{tabular}{|c|c|c|c|}
\hline Variable & OR & $95 \% \mathrm{Cl}$ & $p$ value \\
\hline PVD & 1.35 & $1.14-1.60$ & $<0.001$ \\
\hline Age (per year) & 1.01 & $1.00-1.01$ & $<0.001$ \\
\hline Diabetes & 1.38 & $1.23-1.54$ & $<0.001$ \\
\hline PHx CABGs & 1.47 & $1.15-1.89$ & 0.002 \\
\hline Hypertension & 1.29 & $1.14-1.44$ & $<0.001$ \\
\hline Reference vessel $<2.5 \mathrm{~mm}$ & 1.45 & $1.29-1.62$ & $<0.001$ \\
\hline LAD lesion & 1.19 & $1.07-1.32$ & 0.001 \\
\hline $\mathrm{PHx} \mathrm{MI}$ & 1.24 & $1.12-1.39$ & $<0.001$ \\
\hline B2/C lesion & 1.52 & $1.37-1.69$ & $<0.001$ \\
\hline Ostial lesion & 1.38 & $1.17-1.63$ & $<0.001$ \\
\hline \multicolumn{4}{|l|}{ eGFR } \\
\hline$<30$ & 3.09 & $2.48-3.85$ & $<0.001$ \\
\hline $30-59$ & 1.21 & $1.08-1.37$ & 0.002 \\
\hline \multicolumn{4}{|l|}{ LVEF } \\
\hline$<30 \%$ & 2.61 & $2.05-3.32$ & $<0.001$ \\
\hline $30-45 \%$ & 1.44 & $1.29-1.60$ & $<0.001$ \\
\hline Cardiogenic shock & 5.11 & $4.14-6.30$ & $<0.001$ \\
\hline Thrombolysis & 0.68 & $0.52-0.88$ & 0.003 \\
\hline DES & 0.48 & $0.43-0.53$ & $<0.001$ \\
\hline
\end{tabular}




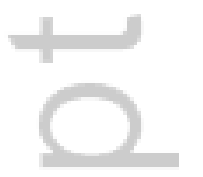

This article is protected by copyright. All rights reserved. 
Table 7. Predictors of 12-Month Mortality

\begin{tabular}{|c|c|c|c|}
\hline Variable & OR & $95 \% \mathrm{Cl}$ & $p$ value \\
\hline PVD & 2.01 & $1.56-2.59$ & $<0.001$ \\
\hline Age & 1.04 & $1.03-1.05$ & $<0.001$ \\
\hline MVD & 1.29 & $1.06-1.58$ & 0.013 \\
\hline Diabetes & 1.44 & $1.19-1.75$ & $<0.001$ \\
\hline Urgent or Rescue $\mathrm{PCl}$ & 2.23 & $1.72-2.88$ & $<0.001$ \\
\hline CLD & 1.58 & $1.25-2.01$ & $<0.001$ \\
\hline Dyslipidaemia & 0.67 & $0.55-0.81$ & $<0.001$ \\
\hline$C D$ & 1.40 & $1.07-1.84$ & 0.014 \\
\hline DES & 0.66 & $0.54-0.80$ & $<0.001$ \\
\hline B2/C lesion & 1.39 & $1.14-1.69$ & 0.001 \\
\hline \multicolumn{4}{|l|}{ eGFR } \\
\hline$<30$ & 5.44 & $4.03-7.34$ & $<0.001$ \\
\hline $30-59$ & 1.85 & $1.51-2.26$ & $<0.001$ \\
\hline \multicolumn{4}{|l|}{ LVEF } \\
\hline$<30 \%$ & 5.23 & $3.83-7.14$ & $<0.001$ \\
\hline $30-45 \%$ & 1.82 & $1.50-2.20$ & $<0.001$ \\
\hline
\end{tabular}




\begin{tabular}{lccc}
\hline Reference vessel $<2.5 \mathrm{~mm}$ & 1.35 & $1.10-1.65$ & 0.003 \\
Cardiogenic shock & 9.18 & $7.14-11.80$ & $<0.001$ \\
RCA lesion & 0.70 & $0.57-0.86$ & 0.001 \\
\hline
\end{tabular}

Table 8. Baseline characteristics in PVD patients with BMS vs. DES

\begin{tabular}{|c|c|c|c|}
\hline & $\begin{array}{l}\text { PVD + BMS } \\
(N=595)\end{array}$ & $\begin{array}{c}\text { PVD + DES } \\
(N=542)\end{array}$ & $\begin{array}{c}\text { p } \\
\text { value }\end{array}$ \\
\hline Mean age - years & $72 \pm 11$ & $70 \pm 10$ & $<0.001$ \\
\hline Male gender & $442(74.3 \%)$ & $402(74.2 \%)$ & 0.96 \\
\hline \multicolumn{4}{|l|}{ Medical history } \\
\hline Diabetes mellitus & $209(35.1 \%)$ & $294(54.2 \%)$ & $<0.001$ \\
\hline Smoking (current) & $112(19.0 \%)$ & $121(22.5 \%)$ & 0.134 \\
\hline Chronic lung disease & $121(20.4 \%)$ & $83(15.4 \%)$ & 0.029 \\
\hline Hypertension & $501(84.3 \%)$ & $486(89.7 \%)$ & 0.008 \\
\hline Dyslipidaemia & $496(83.4 \%)$ & $479(88.5 \%)$ & 0.012 \\
\hline Family history of CAD & $201(34.5 \%)$ & $218(41.1 \%)$ & 0.023 \\
\hline Cerebrovascular disease & $139(23.4 \%)$ & $117(21.6 \%)$ & 0.465 \\
\hline Heart failure & $71(12.0 \%)$ & $65(12.0 \%)$ & 0.984 \\
\hline Previous MI & $258(43.4 \%)$ & $273(50.4 \%)$ & 0.018 \\
\hline Previous $\mathrm{PCl}$ & $161(27.1 \%)$ & $221(40.8 \%)$ & $<0.001$ \\
\hline Previous CABG & $118(19.8 \%)$ & $149(27.5 \%)$ & 0.002 \\
\hline
\end{tabular}




\begin{tabular}{|c|c|c|c|}
\hline eGFR & & & 0.060 \\
\hline$<30$ & $73(12.5 \%)$ & $71(13.2 \%)$ & \\
\hline $30-59$ & $207(35.4 \%)$ & $155(28.8 \%)$ & \\
\hline$>60$ & 305 (52.1\%) & $312(58.0 \%)$ & \\
\hline Mean Baseline Serum Cr & $138.6 \pm 135.8$ & $138.3 \pm 142.5$ & 0.969 \\
\hline Mean BMI $\left(\mathrm{kg} / \mathrm{m}^{2}\right)$ & $27.3(26.9-27.8)$ & $28.2(27.7-28.7)$ & 0.009 \\
\hline \multicolumn{4}{|l|}{ LVEF } \\
\hline$<30 \%$ & $25(5.0 \%)$ & $23(4.9 \%)$ & 0.437 \\
\hline $30-45 \%$ & $159(32.1 \%)$ & $132(28.3 \%)$ & \\
\hline$>45 \%$ & $312(62.9 \%)$ & $311(66.7 \%)$ & \\
\hline \multicolumn{4}{|l|}{ Indication for index $\mathrm{PCl}$} \\
\hline STEMI & $145(24.4 \%)$ & $51(9.4 \%)$ & \\
\hline NSTEMI & $183(30.8 \%)$ & $201(37.1 \%)$ & \\
\hline Unstable angina & $51(8.6 \%)$ & $65(12.0 \%)$ & \\
\hline Non-ACS & $215(36.2 \%)$ & $225(41.5 \%)$ & \\
\hline Out of hospital cardiac arrest & $15(2.5 \%)$ & $3(0.6 \%)$ & 0.008 \\
\hline Cardiogenic shock & $35(5.9 \%)$ & $10(1.9 \%)$ & $<0.001$ \\
\hline IABP & $19(3.2 \%)$ & $5(0.9 \%)$ & 0.008 \\
\hline \multicolumn{4}{|l|}{ Medications } \\
\hline Thrombolytics & $15(2.5 \%)$ & $6(1.1 \%)$ & 0.077 \\
\hline Ilb/IIla Blockade & 139 (23.4\%) & $104(19.3 \%)$ & 0.096 \\
\hline Heparin & $586(98.5 \%)$ & $530(97.8 \%)$ & 0.380 \\
\hline
\end{tabular}




\begin{tabular}{lccc}
\hline LMWH & $120(20.2 \%)$ & $101(18.7 \%)$ & 0.524 \\
Bivalirudin & $9(1.6 \%)$ & $3(0.6 \%)$ & 0.116 \\
Aspirin & $590(99.2 \%)$ & $535(98.7 \%)$ & 0.457 \\
Clopidogrel & $573(96.5 \%)$ & $518(95.8 \%)$ & 0.533 \\
Ticagrelor & $10(1.7 \%)$ & $16(3.0 \%)$ & 0.408 \\
Prasugrel & $9(1.5 \%)$ & $12(2.2 \%)$ & 0.652 \\
\hline
\end{tabular}


Table 9. Angiographic characteristics in PVD patients with BMS vS. DES

\begin{tabular}{|c|c|c|c|}
\hline & $\begin{array}{c}\text { PVD + BMS } \\
(N=595)\end{array}$ & $\begin{array}{l}\text { PVD + DES } \\
(N=542)\end{array}$ & $\begin{array}{c}p \\
\text { value }\end{array}$ \\
\hline \multicolumn{4}{|l|}{ Procedure status } \\
\hline Elective & $217(36.5 \%)$ & $232(42.8 \%)$ & 0.056 \\
\hline Urgent & $375(63.0 \%)$ & $305(56.3 \%)$ & \\
\hline Rescue & $3(0.5 \%)$ & $5(0.9 \%)$ & \\
\hline Percutaneous entry location & & & 0.890 \\
\hline Femoral & $512(86.1 \%)$ & $469(86.5 \%)$ & \\
\hline Radial & $66(11.1 \%)$ & $60(11.1 \%)$ & \\
\hline Disease extent & & & 0.040 \\
\hline Single-vessel disease & $156(26.3 \%)$ & $114(21.1 \%)$ & \\
\hline Multi-vessel disease & $437(73.7 \%)$ & $426(78.9 \%)$ & \\
\hline \multicolumn{4}{|l|}{ Culprit vessel } \\
\hline Left main coronary artery & $17(2.5 \%)$ & $28(4.1 \%)$ & 0.096 \\
\hline Proximal LAD & $105(15.4 \%)$ & $72(10.6 \%)$ & 0.008 \\
\hline LAD & $193(28.3 \%)$ & $188(27.6 \%)$ & 0.750 \\
\hline LCx & $100(14.7 \%)$ & $110(16.1 \%)$ & 0.460 \\
\hline $\mathrm{RCA}$ & $249(36.6 \%)$ & $186(27.3 \%)$ & $<0.001$ \\
\hline Grafts & $54(7.9 \%)$ & $79(11.6 \%)$ & 0.023 \\
\hline Type of coronary lesion & & & $<0.001$ \\
\hline
\end{tabular}




\begin{tabular}{lccc}
\hline De novo & $665(97.7 \%)$ & $585(85.8 \%)$ & \\
Restenosis (no prior stent) & $3(0.4 \%)$ & $4(0.6 \%)$ & \\
In stent restenosis & $13(1.9 \%)$ & $93(13.6 \%)$ & \\
\hline Mean no of lesions & $1.2 \pm 0.4$ & $1.3 \pm 0.5$ & 0.046 \\
treated/patient & & & \\
\hline \multicolumn{1}{c}{ nean of stents } & $1.3 \pm 0.6$ & $1.4 \pm 0.6$ & $<0.001$ \\
deployed/procedure & & & \\
\hline Lesion type & $355(52.1 \%)$ & $403(59.1 \%)$ & 0.010 \\
B2 and C & $3.1 \pm 0.5$ & $2.8 \pm 0.4$ & $<0.001$ \\
\hline Mean stent diameter (mm\%) & $15.7 \pm 4.3$ & $18.1 \pm 6.0$ & $<0.001$ \\
\hline Mean stent length (mm\%) & $48(7.1 \%)$ & $75(11.0 \%)$ & 0.011 \\
\hline Bifurcation lesion & $51(7.5 \%)$ & $70(10.3 \%)$ & 0.072 \\
\hline Ostial lesion & $644(99.4 \%)$ & $636(98.0 \%)$ & 0.028 \\
\hline Procedural success rate & & & \\
\hline
\end{tabular}


Table 10. 30 Day Medication Use in PVD patients with BMS vs. DES

\begin{tabular}{lccc}
\hline & PVD + BMS & PVD + DES & p \\
& (N = 595) & $(\mathbf{N}=542)$ & value \\
\hline Antiplatelet/Anticoagulant & & & \\
No AP and no AC & $3(0.6 \%)$ & $0(0.0 \%)$ & 0.088 \\
Aspirin only & $25(4.9 \%)$ & $4(0.8 \%)$ & $<0.001$ \\
Thienopyridine only & $10(2.0 \%)$ & $10(2.0 \%)$ & 0.939 \\
Aspirin and Thienopyridine & $457(89.3 \%)$ & $472(95.2 \%)$ & $<0.001$ \\
Anticoagulation only & $4(0.8 \%)$ & $1(0.2 \%)$ & 0.191 \\
Single AP and AC & $13(2.6 \%)$ & $9(1.8 \%)$ & 0.434 \\
Triple therapy & $59(11.6 \%)$ & $13(2.6 \%)$ & $<0.001$ \\
\hline Statin & $461(90.8 \%)$ & $453(91.9)$ & 0.523 \\
Fibrate & $17(3.9 \%)$ & $7(1.7 \%)$ & 0.056 \\
Ezetimibe & $36(8.2 \%)$ & $50(12.2 \%)$ & 0.058 \\
Beta Blocker & $351(69.6 \%)$ & $356(72.4 \%)$ & 0.345 \\
ACE inhibitor & $271(54.0 \%)$ & $278(56.5 \%)$ & 0.424 \\
ARB & $130(25.8 \%)$ & $139(28.3 \%)$ & 0.372 \\
Nitrate & $97(22.4 \%)$ & $85(20.7 \%)$ & 0.556 \\
Ca Channel Blocker & $118(27.3 \%)$ & $120(29.3)$ & 0.529 \\
\hline
\end{tabular}


Table 11. 12-Month Medication Use in PVD patients with BMS vs. DES

\begin{tabular}{lccc}
\hline & PVD + BMS & PVD + DES & p \\
& (N = 595) & (N = 542) & value \\
\hline Antiplatelet/Anticoagulant & & & \\
No AP and no AC & $10(2.3 \%)$ & $2(0.5 \%)$ & 0.018 \\
Aspirin only & $146(33.3 \%)$ & $57(12.7 \%)$ & $<0.001$ \\
Thienopyridine only & $13(3.0 \%)$ & $21(4.7 \%)$ & 0.183 \\
Aspirin and Thienopyridine & $226(51.4 \%)$ & $360(79.8 \%)$ & $<0.001$ \\
Anticoagulation only & $7(1.6 \%)$ & $3(0.7 \%)$ & 0.191 \\
Single AP and AC & $37(8.5 \%)$ & $6(1.3 \%)$ & $<0.001$ \\
Triple therapy & $22(5.0 \%)$ & $10(2.2 \%)$ & 0.026 \\
\hline Statin & $398(90.7 \%)$ & $407(90.9)$ & 0.923 \\
Fibrate & $17(4.0 \%)$ & $8(1.9 \%)$ & 0.069 \\
Ezetimibe & $41(9.7 \%)$ & $58(13.8 \%)$ & 0.067 \\
Beta Blocker & $280(64.5 \%)$ & $298(67.1 \%)$ & 0.417 \\
ACE inhibitor & $217(49.9 \%)$ & $231(52.3 \%)$ & 0.481 \\
ARB & $126(28.9 \%)$ & $132(29.9 \%)$ & 0.754 \\
Nitrate & $67(16.0 \%)$ & $67(16.0 \%)$ & 1.000 \\
Ca Channel Blocker & $125(29.9 \%)$ & $149(35.7)$ & 0.077 \\
\hline
\end{tabular}


Table 12. In-Hospital, 30-Day and 12-Month Outcomes in PVD patients with BMS vs. DES

\begin{tabular}{lccc}
\hline & PVD + BMS & PVD + DES & p value \\
& (N = 595) & (N = 542) & \\
\hline In-Hospital & $38(6.4 \%)$ & $15(2.8 \%)$ & 0.004 \\
MACE & $25(4.2 \%)$ & $10(1.9 \%)$ & 0.022 \\
Death & $12(2.0 \%)$ & $1(0.2 \%)$ & 0.004 \\
New or recurrent MI & $1(0.2 \%)$ & $2(0.4 \%)$ & 0.507 \\
Repeat PCl & $4(0.7 \%)$ & $1(0.2 \%)$ & 0.215 \\
CABGs & $13(2.2 \%)$ & $6(1.1 \%)$ & 0.158 \\
New Renal Impairment & $22(3.7 \%)$ & $20(3.7 \%)$ & 0.995 \\
Bleeding event & & & 0.721 \\
Site of bleeding & $2(9.5 \%)$ & $2(10.0 \%)$ & \\
$\quad$ Retroperitoneal & $6(28.6 \%)$ & $8(40.0 \%)$ & \\
Percutaneous entry site & $13(61.9 \%)$ & $10(50.0 \%)$ & \\
Other & & & \\
Vascular Complication & 0 & 0 & \\
Access Site Occlusion & & & \\
\hline
\end{tabular}




\begin{tabular}{|c|c|c|c|}
\hline Loss of distal pulse & $0(0.0 \%)$ & $1(0.2 \%)$ & 0.295 \\
\hline Peripheral Arterial Dissection & $4(0.7 \%)$ & $3(0.6 \%)$ & 0.158 \\
\hline AV Fistula & $1(0.2 \%)$ & $0(0.0 \%)$ & 0.340 \\
\hline Pseudoaneurysm & $0(0.0 \%)$ & $1(0.2 \%)$ & 0.295 \\
\hline \multicolumn{4}{|l|}{ 30-Days } \\
\hline MACE & $60(10.1 \%)$ & $26(4.8 \%)$ & 0.001 \\
\hline Death & $35(5.9 \%)$ & $14(2.6 \%)$ & 0.006 \\
\hline Cardiac cause & $25(71.4 \%)$ & $11(78.6 \%)$ & 0.609 \\
\hline Myocardial infarction & $25(4.2 \%)$ & $9(1.7 \%)$ & 0.012 \\
\hline TVR & $13(2.2 \%)$ & $8(1.5 \%)$ & 0.375 \\
\hline \multicolumn{4}{|l|}{ 12-Months } \\
\hline MACE & $157(26.4 \%)$ & $105(19.4 \%)$ & 0.005 \\
\hline Death & $87(14.6 \%)$ & $46(8.5 \%)$ & 0.001 \\
\hline Cardiac cause & $44(50.6 \%)$ & $30(65.2 \%)$ & 0.106 \\
\hline Myocardial infarction & $61(10.3 \%)$ & $44(8.1 \%)$ & 0.214 \\
\hline TVR & $51(8.6 \%)$ & $46(8.5 \%)$ & 0.959 \\
\hline
\end{tabular}




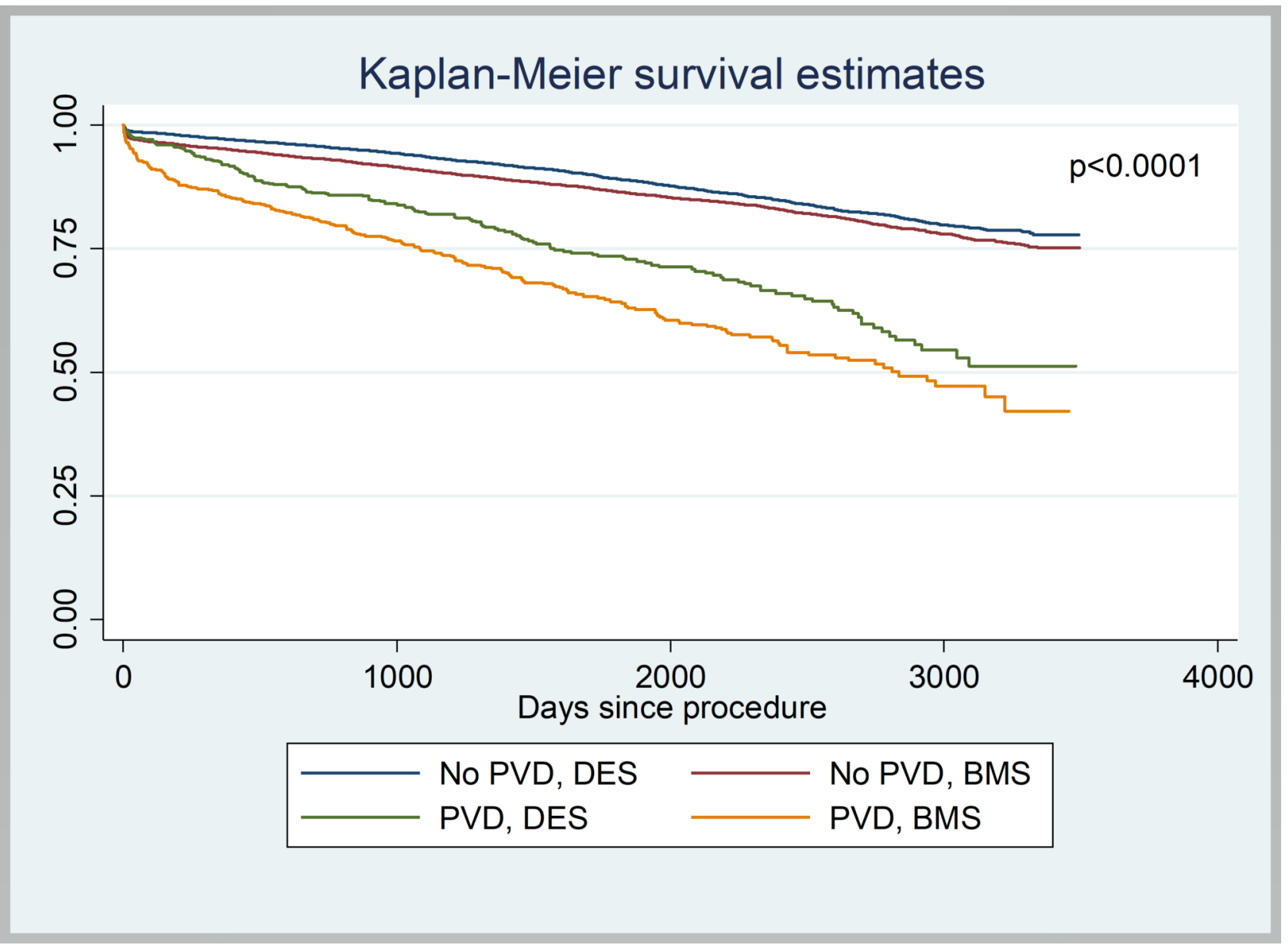

CCD_28145_figure 1_300.tiff

This article is protected by copyright. All rights reserved. 
Table 1: Baseline characteristics

\begin{tabular}{|c|c|c|c|}
\hline & $\begin{array}{c}\text { PVD } \\
(\mathrm{N}=1,251)\end{array}$ & $\begin{array}{c}\text { No PVD } \\
(\mathrm{N}=17,129)\end{array}$ & $\begin{array}{c}\text { p } \\
\text { value }\end{array}$ \\
\hline Mean age - years & $71.0 \pm 10$ & $64 \pm 12$ & $<0.001$ \\
\hline Male gender & $928(74.2 \%)$ & $12,956(75.6 \%)$ & 0.25 \\
\hline \multicolumn{4}{|l|}{ Medical history } \\
\hline Diabetes mellitus & 564 (45.1\%) & $3,998(23.4 \%)$ & $<0.001$ \\
\hline Smoking (current) & $244(19.7 \%)$ & $4,137(24.3 \%)$ & $<0.001$ \\
\hline Chronic lung disease & $219(17.6 \%)$ & $1,627(9.5 \%)$ & $<0.001$ \\
\hline Hypertension & $1,088(87.0 \%)$ & $11,206(65.4 \%)$ & $<0.001$ \\
\hline Dyslipidaemia & $1,076(86.1 \%)$ & $11,905(69.6 \%)$ & $<0.001$ \\
\hline Family history of CAD & $464(37.9 \%)$ & 6,596 (38.9\%) & 0.524 \\
\hline Cerebrovascular disease & $282(22.6 \%)$ & $943(5.5 \%)$ & $<0.001$ \\
\hline Heart failure & $154(12.32 \%)$ & $557(3.3 \%)$ & $<0.001$ \\
\hline Previous MI & $608(48.6 \%)$ & 4,495 (26.3\%) & $<0.001$ \\
\hline Previous $\mathrm{PCl}$ & $441(35.3 \%)$ & $4,221(24.65 \%)$ & $<0.001$ \\
\hline Previous CABG & $311(24.9 \%)$ & $1,308(7.6 \%)$ & $<0.001$ \\
\hline eGFR & & & $<0.001$ \\
\hline$<30$ & $163(13.2 \%)$ & $400(2.4 \%)$ & \\
\hline $30-59$ & $397(32.2 \%)$ & $3,273(19.6 \%)$ & \\
\hline$>60$ & $675(54.7 \%)$ & 13,065 (78.1\%) & \\
\hline Mean Baseline Serum Cr & $140.8 \pm 144.7$ & $93.2 \pm 66.3$ & $<0.001$ \\
\hline Mean BMI $\left(\mathrm{kg} / \mathrm{m}^{2}\right)$ & $27.8(27.4-28.1)$ & $28.4(28.3-28.5)$ & $<0.001$ \\
\hline \multicolumn{4}{|l|}{ LVEF } \\
\hline$<30 \%$ & $53(5.0 \%)$ & $350(2.35 \%)$ & $<0.001$ \\
\hline
\end{tabular}




\begin{tabular}{|c|c|c|c|}
\hline $30-45 \%$ & $325(30.8 \%)$ & $3,384(22.7 \%)$ & \\
\hline$>45 \%$ & $677(64.2 \%)$ & $11,172(75.0 \%)$ & \\
\hline \multicolumn{4}{|l|}{ Indication for index $\mathrm{PCl}$} \\
\hline STEMI & $217(17.4 \%)$ & $5,249(30.7 \%)$ & \\
\hline NSTEMI & $413(33.0 \%)$ & $4,503(26.3 \%)$ & \\
\hline Unstable angina & $127(10.2 \%)$ & $1,669(9.8 \%)$ & \\
\hline Non-ACS & $493(39.4 \%)$ & $5,696(33.3 \%)$ & \\
\hline Out of hospital cardiac arrest & $21(1.7 \%)$ & $437(2.6 \%)$ & 0.056 \\
\hline Cardiogenic shock & $52(4.2 \%)$ & $550(3.2 \%)$ & 0.070 \\
\hline IABP & $27(2.2 \%)$ & $422(2.5 \%)$ & 0.50 \\
\hline \multicolumn{4}{|l|}{ Medications } \\
\hline Thrombolytics & $22(1.8 \%)$ & $801(4.7 \%)$ & $<0.001$ \\
\hline IIb/IIla Blockade & $263(21.1 \%)$ & $5,399(31.5 \%)$ & $<0.001$ \\
\hline Heparin & $1229(98.2 \%)$ & $16726(97.7 \%)$ & 0.21 \\
\hline LMWH & $239(19.1 \%)$ & $3272(19.1 \%)$ & 0.99 \\
\hline Bivalirudin & $13(1.1 \%)$ & $258(1.6 \%)$ & 0.15 \\
\hline Aspirin & $1239(99.0 \%)$ & $16900(98.7 \%)$ & 0.28 \\
\hline Clopidogrel & 1167 (93.4\%) & $15918(93.0 \%)$ & 0.55 \\
\hline
\end{tabular}




\begin{tabular}{|c|c|c|c|}
\hline & $\begin{array}{c}\text { PVD } \\
(N=1,251)\end{array}$ & $\begin{array}{c}\text { No PVD } \\
(\mathrm{N}=17,129)\end{array}$ & $\begin{array}{c}\text { p } \\
\text { value }\end{array}$ \\
\hline \multicolumn{4}{|l|}{ Procedure status } \\
\hline Elective & $502(40.3 \%)$ & $5,866(34.3 \%)$ & $<0.001$ \\
\hline Urgent & $739(59.1 \%)$ & $10,985(64.1 \%)$ & \\
\hline Rescue & $10(0.8 \%)$ & $278(1.6 \%)$ & \\
\hline Percutaneous entry location & & & $<0.001$ \\
\hline Femoral & $1,079(86.3 \%)$ & $15,412(90.0 \%)$ & \\
\hline Radial & $137(11.0 \%)$ & $1,671(9.8 \%)$ & \\
\hline Disease extent & & & $<0.001$ \\
\hline Single-vessel disease & $293(23.5 \%)$ & $7,086(41.6 \%)$ & \\
\hline Multi-vessel disease & $954(76.5 \%)$ & $9,963(58.4 \%)$ & \\
\hline \multicolumn{4}{|l|}{ Culprit vessel } \\
\hline Left main coronary artery & $51(3.4 \%)$ & $170(0.8 \%)$ & $<0.001$ \\
\hline Proximal LAD & $193(12.7 \%)$ & $3,302(16.3 \%)$ & $<0.001$ \\
\hline LAD & $415(27.4 \%)$ & $7,057(34.8 \%)$ & $<0.001$ \\
\hline LCX & $228(15.1 \%)$ & $2,668(13.2 \%)$ & 0.036 \\
\hline $\mathrm{RCA}$ & $480(31.7 \%)$ & $6,496(32.1 \%)$ & 0.77 \\
\hline Grafts & $145(9.6 \%)$ & $491(2.4 \%)$ & $<0.001$ \\
\hline \multicolumn{4}{|l|}{ Type of coronary lesion } \\
\hline De novo & $1,362(89.9 \%)$ & $19,034(93.9 \%)$ & $<0.001$ \\
\hline Restenosis (no prior stent) & $9(0.6 \%)$ & $66(0.3 \%)$ & \\
\hline In stent restenosis & $144(9.5 \%)$ & $1,165(5.8 \%)$ & \\
\hline Mean no of lesions & $1.2 \pm 0.5$ & $1.2 \pm 0.4$ & 0.02 \\
\hline
\end{tabular}




\begin{tabular}{lccc}
\hline \multicolumn{1}{c}{ treated/patient } & & & \\
\hline Mean no. of stents & $1.2 \pm 0.7$ & $1.2 \pm 0.6$ & 0.90 \\
deployed/procedure & & & \\
\hline Lesion type & & & \\
B2 and C & $869(57.4 \%)$ & $10,960(54.1 \%)$ & 0.013 \\
\hline Mean stent diameter (mm\%) & $2.9 \pm 0.5$ & $2.9 \pm 0.5$ & 0.20 \\
\hline Mean stent length (mm\%) & $16.9 \pm 5.3$ & $17.3 \pm 5.6$ & 0.009 \\
\hline Bifurcation lesion & $142(9.4 \%)$ & $2,197(10.8 \%)$ & 0.075 \\
\hline Ostial lesion & $142(9.4 \%)$ & $1,368(6.8 \%)$ & $<0.001$ \\
\hline Type of stent & & & \\
Balloon only & $114(9.1 \%)$ & $1,066(6.2 \%)$ & $<0.001$ \\
Bare metal & $595(47.6 \%)$ & $8,386(49.0 \%)$ & \\
Drug eluting & $542(43.3 \%)$ & $7,677(44.8 \%)$ & \\
\hline Procedural success rate & & & \\
\hline
\end{tabular}




\begin{tabular}{|c|c|c|c|}
\hline & $\begin{array}{c}\text { PVD } \\
(N=1,090)\end{array}$ & $\begin{array}{c}\text { No PVD } \\
(\mathrm{N}=15,601)\end{array}$ & $\begin{array}{c}\text { p } \\
\text { value }\end{array}$ \\
\hline \multicolumn{4}{|l|}{ Antiplatelet/Anticoagulant } \\
\hline No AP and no AC & $4(0.4 \%)$ & $34(0.2 \%)$ & 0.318 \\
\hline Aspirin only & $52(4.8 \%)$ & $593(3.8 \%)$ & 0.108 \\
\hline Thienopyridine only & $22(2.0 \%)$ & $193(1.2 \%)$ & 0.027 \\
\hline Aspirin and Thienopyridine & $985(89.6 \%)$ & $14,673(93.2 \%)$ & $<0.001$ \\
\hline Anticoagulation only & $6(0.6 \%)$ & $19(0.1 \%)$ & $<0.001$ \\
\hline Single AP and AC & $30(2.8 \%)$ & $213(1.4 \%)$ & $<0.001$ \\
\hline Triple therapy & $78(7.2 \%)$ & $813(5.2 \%)$ & 0.006 \\
\hline Statin & $998(91.6 \%)$ & $14,816(94.7)$ & $<0.001$ \\
\hline Fibrate & $24(2.6 \%)$ & $224(1.8 \%)$ & 0.070 \\
\hline Ezetimibe & $100(10.8 \%)$ & $586(4.6 \%)$ & $<0.001$ \\
\hline Beta Blocker & $774(71.3 \%)$ & $12,142(77.9 \%)$ & $<0.001$ \\
\hline ACE inhibitor & $599(55.3 \%)$ & $9,942(63.8 \%)$ & $<0.001$ \\
\hline ARB & $287(26.5 \%)$ & $2,722(17.5 \%)$ & $<0.001$ \\
\hline Nitrate & $200(21.8 \%)$ & 1, $056(8.4 \%)$ & $<0.001$ \\
\hline Ca Channel Blocker & $268(29.1 \%)$ & 1, $975(15.7)$ & $<0.001$ \\
\hline
\end{tabular}




\begin{tabular}{|c|c|c|c|}
\hline & $\begin{array}{c}\text { PVD } \\
(\mathrm{N}=966)\end{array}$ & $\begin{array}{c}\text { No PVD } \\
(\mathrm{N}=14,696)\end{array}$ & $\begin{array}{c}\text { p } \\
\text { value }\end{array}$ \\
\hline \multicolumn{4}{|l|}{ Antiplatelet/Anticoagulant } \\
\hline No AP and no AC & $13(1.4 \%)$ & $167(1.1 \%)$ & 0.528 \\
\hline Aspirin only & $231(23.9 \%)$ & 3, $890(26.1 \%)$ & 0.121 \\
\hline Thienopyridine only & $39(4.0 \%)$ & $493(3.3 \%)$ & 0.230 \\
\hline Aspirin and Thienopyridine & $619(63.7 \%)$ & $9,785(65.6 \%)$ & 0.233 \\
\hline Anticoagulation only & $14(1.5 \%)$ & $47(0.3 \%)$ & 0.000 \\
\hline Single AP and AC & $52(5.4 \%)$ & $520(3.5 \%)$ & 0.002 \\
\hline Triple therapy & $34(3.5 \%)$ & $377(2.5 \%)$ & 0.006 \\
\hline Statin & $882(91.2 \%)$ & $13,828(92.7)$ & 0.079 \\
\hline Fibrate & $25(2.7 \%)$ & $291(2.1 \%)$ & 0.211 \\
\hline Ezetimibe & $114(12.4 \%)$ & $943(6.8 \%)$ & $<0.001$ \\
\hline Beta Blocker & $630(65.8 \%)$ & $10,564(71.3 \%)$ & $<0.001$ \\
\hline ACE inhibitor & $490(51.3 \%)$ & $8,718(58.9 \%)$ & $<0.001$ \\
\hline ARB & $276(28.8 \%)$ & $3,154(21.3 \%)$ & $<0.001$ \\
\hline Nitrate & $153(16.7 \%)$ & $1,160(8.4 \%)$ & $<0.001$ \\
\hline Ca Channel Blocker & $302(33.2 \%)$ & 2, $406(17.5)$ & $<0.001$ \\
\hline
\end{tabular}


Table 5. In-Hospital, 30-Day and 12-Month Clinical Outcomes

\begin{tabular}{|c|c|c|c|}
\hline & $\begin{array}{c}\text { PVD } \\
(N=1,251)\end{array}$ & $\begin{array}{c}\text { No PVD } \\
(\mathrm{N}=17,129)\end{array}$ & $p$ value \\
\hline \multicolumn{4}{|l|}{ In-Hospital } \\
\hline MACE & $71(5.7 \%)$ & $705(4.1 \%)$ & 0.008 \\
\hline Death & $47(3.8 \%)$ & $357(2.1 \%)$ & $<0.001$ \\
\hline New or recurrent MI & $17(1.4 \%)$ & $199(1.2 \%)$ & 0.534 \\
\hline Repeat PCl & $3(0.2 \%)$ & $108(0.6 \%)$ & 0.084 \\
\hline CABGs & $10(0.8 \%)$ & $148(0.9 \%)$ & 0.806 \\
\hline New Renal Impairment & $24(1.9 \%)$ & $196(1.1 \%)$ & 0.015 \\
\hline Bleeding event & $47(3.8 \%)$ & $418(2.4 \%)$ & 0.004 \\
\hline Site of bleeding & & & 0.698 \\
\hline Retroperitoneal & $4(8.7 \%)$ & $24(6 \%)$ & \\
\hline Percutaneous entry site & $16(34.8 \%)$ & $157(39.4 \%)$ & \\
\hline Other & $26(56.5 \%)$ & $218(54.6 \%)$ & \\
\hline \multicolumn{4}{|l|}{ Vascular Complication } \\
\hline Access Site Occlusion & 0 & $6(0.04 \%)$ & 0.501 \\
\hline Loss of distal pulse & $1(0.1 \%)$ & $17(0.1 \%)$ & 0.833 \\
\hline Peripheral Arterial Dissection & $7(0.6 \%)$ & $29(0.2 \%)$ & 0.003 \\
\hline AV Fistula & $1(0.1 \%)$ & $9(0.1 \%)$ & 0.688 \\
\hline Pseudoaneurysm & $1(0.1 \%)$ & $56(0.3 \%)$ & 0.129 \\
\hline \multicolumn{4}{|l|}{ 30-Days } \\
\hline MACE & $107(8.6 \%)$ & $996(5.8 \%)$ & $<0.001$ \\
\hline Death & $62(5.0 \%)$ & $416(2.4 \%)$ & $<0.001$ \\
\hline Cardiac cause & $48(77.4 \%)$ & $329(79.3 \%)$ & 0.737 \\
\hline
\end{tabular}




\begin{tabular}{lccc}
\hline Myocardial infarction & $39(3.12 \%)$ & $339(2.0 \%)$ & 0.006 \\
TVR & $27(2.2 \%)$ & $418(2.4 \%)$ & 0.531 \\
\hline 12-Months & $308(24.6 \%)$ & $2264(13.2 \%)$ & $<0.001$ \\
MACE & $153(12.2 \%)$ & $720(4.2 \%)$ & $<0.001$ \\
Death & $90(58.8 \%)$ & $431(60.1 \%)$ & 0.768 \\
$\quad$ Cardiac cause & $123(9.8 \%)$ & $758(4.4 \%)$ & $<0.001$ \\
Myocardial infarction & $119(9.5 \%)$ & $1236(7.2 \%)$ & 0.003 \\
TVR & & & \\
\hline
\end{tabular}

This article is protected by copyright. All rights reserved. 
Table 6. Predictors of 12-Month MACE

\begin{tabular}{|c|c|c|c|}
\hline Variable & OR & $95 \% \mathrm{Cl}$ & p value \\
\hline PVD & 1.35 & $1.14-1.60$ & $<0.001$ \\
\hline Age (per year) & 1.01 & $1.00-1.01$ & $<0.001$ \\
\hline Diabetes & 1.38 & $1.23-1.54$ & $<0.001$ \\
\hline PHx CABGs & 1.47 & $1.15-1.89$ & 0.002 \\
\hline Hypertension & 1.29 & $1.14-1.44$ & $<0.001$ \\
\hline Reference vessel $<2.5 \mathrm{~mm}$ & 1.45 & $1.29-1.62$ & $<0.001$ \\
\hline LAD lesion & 1.19 & $1.07-1.32$ & 0.001 \\
\hline $\mathrm{PHx} \mathrm{MI}$ & 1.24 & $1.12-1.39$ & $<0.001$ \\
\hline B2/C lesion & 1.52 & $1.37-1.69$ & $<0.001$ \\
\hline Ostial lesion & 1.38 & $1.17-1.63$ & $<0.001$ \\
\hline \multicolumn{4}{|l|}{ eGFR } \\
\hline$<30$ & 3.09 & $2.48-3.85$ & $<0.001$ \\
\hline $30-59$ & 1.21 & $1.08-1.37$ & 0.002 \\
\hline \multicolumn{4}{|l|}{ LVEF } \\
\hline$<30 \%$ & 2.61 & $2.05-3.32$ & $<0.001$ \\
\hline $30-45 \%$ & 1.44 & $1.29-1.60$ & $<0.001$ \\
\hline Cardiogenic shock & 5.11 & $4.14-6.30$ & $<0.001$ \\
\hline Thrombolysis & 0.68 & $0.52-0.88$ & 0.003 \\
\hline DES & 0.48 & $0.43-0.53$ & $<0.001$ \\
\hline
\end{tabular}


Table 7. Predictors of 12-Month Mortality

\begin{tabular}{|c|c|c|c|}
\hline Variable & OR & $95 \% \mathrm{Cl}$ & $p$ value \\
\hline PVD & 2.01 & $1.56-2.59$ & $<0.001$ \\
\hline Age & 1.04 & $1.03-1.05$ & $<0.001$ \\
\hline MVD & 1.29 & $1.06-1.58$ & 0.013 \\
\hline Diabetes & 1.44 & $1.19-1.75$ & $<0.001$ \\
\hline Urgent or Rescue PCl & 2.23 & $1.72-2.88$ & $<0.001$ \\
\hline CLD & 1.58 & $1.25-2.01$ & $<0.001$ \\
\hline Dyslipidaemia & 0.67 & $0.55-0.81$ & $<0.001$ \\
\hline CD & 1.40 & $1.07-1.84$ & 0.014 \\
\hline DES & 0.66 & $0.54-0.80$ & $<0.001$ \\
\hline B2/C lesion & 1.39 & $1.14-1.69$ & 0.001 \\
\hline \multicolumn{4}{|l|}{ eGFR } \\
\hline$<30$ & 5.44 & $4.03-7.34$ & $<0.001$ \\
\hline $30-59$ & 1.85 & $1.51-2.26$ & $<0.001$ \\
\hline \multicolumn{4}{|l|}{ LVEF } \\
\hline$<30 \%$ & 5.23 & $3.83-7.14$ & $<0.001$ \\
\hline $30-45 \%$ & 1.82 & $1.50-2.20$ & $<0.001$ \\
\hline Reference vessel $<2.5 \mathrm{~mm}$ & 1.35 & $1.10-1.65$ & 0.003 \\
\hline Cardiogenic shock & 9.18 & $7.14-11.80$ & $<0.001$ \\
\hline RCA lesion & 0.70 & $0.57-0.86$ & 0.001 \\
\hline
\end{tabular}


Table 8. Baseline characteristics in PVD patients with BMS vS. DES

\begin{tabular}{|c|c|c|c|}
\hline & $\begin{array}{c}\text { PVD + BMS } \\
(N=595)\end{array}$ & $\begin{array}{c}\text { PVD + DES } \\
(N=542)\end{array}$ & $\begin{array}{c}p \\
\text { value }\end{array}$ \\
\hline Mean age - years & $72 \pm 11$ & $70 \pm 10$ & $<0.001$ \\
\hline Male gender & $442(74.3 \%)$ & $402(74.2 \%)$ & 0.96 \\
\hline \multicolumn{4}{|l|}{ Medical history } \\
\hline Diabetes mellitus & $209(35.1 \%)$ & $294(54.2 \%)$ & $<0.001$ \\
\hline Smoking (current) & $112(19.0 \%)$ & $121(22.5 \%)$ & 0.134 \\
\hline Chronic lung disease & $121(20.4 \%)$ & $83(15.4 \%)$ & 0.029 \\
\hline Hypertension & $501(84.3 \%)$ & $486(89.7 \%)$ & 0.008 \\
\hline Dyslipidaemia & $496(83.4 \%)$ & $479(88.5 \%)$ & 0.012 \\
\hline Family history of CAD & $201(34.5 \%)$ & $218(41.1 \%)$ & 0.023 \\
\hline Cerebrovascular disease & $139(23.4 \%)$ & $117(21.6 \%)$ & 0.465 \\
\hline Heart failure & $71(12.0 \%)$ & $65(12.0 \%)$ & 0.984 \\
\hline Previous MI & $258(43.4 \%)$ & $273(50.4 \%)$ & 0.018 \\
\hline Previous $\mathrm{PCl}$ & $161(27.1 \%)$ & $221(40.8 \%)$ & $<0.001$ \\
\hline Previous CABG & $118(19.8 \%)$ & $149(27.5 \%)$ & 0.002 \\
\hline eGFR & & & 0.060 \\
\hline$<30$ & $73(12.5 \%)$ & $71(13.2 \%)$ & \\
\hline $30-59$ & 207 (35.4\%) & $155(28.8 \%)$ & \\
\hline$>60$ & $305(52.1 \%)$ & $312(58.0 \%)$ & \\
\hline Mean Baseline Serum Cr & $138.6 \pm 135.8$ & $138.3 \pm 142.5$ & 0.969 \\
\hline Mean BMI $\left(\mathrm{kg} / \mathrm{m}^{2}\right)$ & $27.3(26.9-27.8)$ & $28.2(27.7-28.7)$ & 0.009 \\
\hline LVEF & & & 0.437 \\
\hline$<30 \%$ & $25(5.0 \%)$ & $23(4.9 \%)$ & \\
\hline
\end{tabular}




\begin{tabular}{|c|c|c|c|}
\hline $30-45 \%$ & $159(32.1 \%)$ & $132(28.3 \%)$ & \\
\hline$>45 \%$ & $312(62.9 \%)$ & $311(66.7 \%)$ & \\
\hline \multicolumn{4}{|l|}{ Indication for index $\mathrm{PCl}$} \\
\hline STEMI & $145(24.4 \%)$ & $51(9.4 \%)$ & \\
\hline NSTEMI & $183(30.8 \%)$ & $201(37.1 \%)$ & 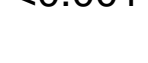 \\
\hline Unstable angina & $51(8.6 \%)$ & $65(12.0 \%)$ & \\
\hline Non-ACS & $215(36.2 \%)$ & $225(41.5 \%)$ & \\
\hline Out of hospital cardiac arrest & $15(2.5 \%)$ & $3(0.6 \%)$ & 0.008 \\
\hline Cardiogenic shock & $35(5.9 \%)$ & $10(1.9 \%)$ & $<0.001$ \\
\hline IABP & $19(3.2 \%)$ & $5(0.9 \%)$ & 0.008 \\
\hline \multicolumn{4}{|l|}{ Medications } \\
\hline Thrombolytics & $15(2.5 \%)$ & $6(1.1 \%)$ & 0.077 \\
\hline IIb/IIla Blockade & $139(23.4 \%)$ & $104(19.3 \%)$ & 0.096 \\
\hline Heparin & $586(98.5 \%)$ & $530(97.8 \%)$ & 0.380 \\
\hline LMWH & $120(20.2 \%)$ & $101(18.7 \%)$ & 0.524 \\
\hline Bivalirudin & $9(1.6 \%)$ & $3(0.6 \%)$ & 0.116 \\
\hline Aspirin & $590(99.2 \%)$ & $535(98.7 \%)$ & 0.457 \\
\hline Clopidogrel & $573(96.5 \%)$ & $518(95.8 \%)$ & 0.533 \\
\hline Ticagrelor & $10(1.7 \%)$ & $16(3.0 \%)$ & 0.408 \\
\hline Prasugrel & $9(1.5 \%)$ & $12(2.2 \%)$ & 0.652 \\
\hline
\end{tabular}


Table 9. Angiographic characteristics in PVD patients with BMS vs. DES

\begin{tabular}{|c|c|c|c|}
\hline & $\begin{array}{c}\text { PVD + BMS } \\
(N=595)\end{array}$ & $\begin{array}{l}\text { PVD + DES } \\
(N=542)\end{array}$ & $\begin{array}{c}\text { p } \\
\text { value }\end{array}$ \\
\hline \multicolumn{4}{|l|}{ Procedure status } \\
\hline Elective & $217(36.5 \%)$ & $232(42.8 \%)$ & 0.056 \\
\hline Urgent & $375(63.0 \%)$ & $305(56.3 \%)$ & \\
\hline Rescue & $3(0.5 \%)$ & $5(0.9 \%)$ & \\
\hline Percutaneous entry location & & & 0.890 \\
\hline Femoral & $512(86.1 \%)$ & $469(86.5 \%)$ & \\
\hline Radial & $66(11.1 \%)$ & $60(11.1 \%)$ & \\
\hline Disease extent & & & 0.040 \\
\hline Single-vessel disease & $156(26.3 \%)$ & $114(21.1 \%)$ & \\
\hline Multi-vessel disease & $437(73.7 \%)$ & $426(78.9 \%)$ & \\
\hline \multicolumn{4}{|l|}{ Culprit vessel } \\
\hline Left main coronary artery & $17(2.5 \%)$ & $28(4.1 \%)$ & 0.096 \\
\hline Proximal LAD & $105(15.4 \%)$ & $72(10.6 \%)$ & 0.008 \\
\hline LAD & $193(28.3 \%)$ & $188(27.6 \%)$ & 0.750 \\
\hline LCx & $100(14.7 \%)$ & $110(16.1 \%)$ & 0.460 \\
\hline $\mathrm{RCA}$ & $249(36.6 \%)$ & $186(27.3 \%)$ & $<0.001$ \\
\hline Grafts & $54(7.9 \%)$ & $79(11.6 \%)$ & 0.023 \\
\hline \multicolumn{4}{|l|}{ Type of coronary lesion } \\
\hline De novo & $665(97.7 \%)$ & $585(85.8 \%)$ & $<0.001$ \\
\hline Restenosis (no prior stent) & $3(0.4 \%)$ & $4(0.6 \%)$ & \\
\hline In stent restenosis & $13(1.9 \%)$ & $93(13.6 \%)$ & \\
\hline Mean no of lesions & $1.2 \pm 0.4$ & $1.3 \pm 0.5$ & 0.046 \\
\hline
\end{tabular}




\begin{tabular}{lccc}
\hline \multicolumn{1}{l}{ treated/patient } & & & \\
\hline Mean no. of stents & $1.3 \pm 0.6$ & $1.4 \pm 0.6$ & $<0.001$ \\
deployed/procedure & & & \\
\hline Lesion type & & & 0.010 \\
B2 and C & $355(52.1 \%)$ & $403(59.1 \%)$ & $<0.001$ \\
\hline Mean stent diameter (mm\%) & $3.1 \pm 0.5$ & $2.8 \pm 0.4$ & 0.011 \\
\hline Mean stent length (mm\%) & $15.7 \pm 4.3$ & $18.1 \pm 6.0$ & 0.072 \\
\hline Bifurcation lesion & $48(7.1 \%)$ & $75(11.0 \%)$ & 0.028 \\
\hline Ostial lesion & $51(7.5 \%)$ & $70(10.3 \%)$ & \\
\hline Procedural success rate & $644(99.4 \%)$ & $636(98.0 \%)$ & \\
\hline
\end{tabular}


Table 10. 30 Day Medication Use in PVD patients with BMS vs. DES

\begin{tabular}{lccc}
\hline & PVD + BMS & PVD + DES & p \\
& (N = 595) & (N = 542) & value \\
\hline Antiplatelet/Anticoagulant & $3(0.6 \%)$ & $0(0.0 \%)$ & 0.088 \\
No AP and no AC & $25(4.9 \%)$ & $4(0.8 \%)$ & $<0.001$ \\
Aspirin only & $10(2.0 \%)$ & $10(2.0 \%)$ & 0.939 \\
Thienopyridine only & $457(89.3 \%)$ & $472(95.2 \%)$ & $<0.001$ \\
Aspirin and Thienopyridine & $4(0.8 \%)$ & $1(0.2 \%)$ & 0.191 \\
Anticoagulation only & $13(2.6 \%)$ & $9(1.8 \%)$ & 0.434 \\
Single AP and AC & $59(11.6 \%)$ & $13(2.6 \%)$ & $<0.001$ \\
Triple therapy & $461(90.8 \%)$ & $453(91.9)$ & 0.523 \\
\hline Statin & $17(3.9 \%)$ & $7(1.7 \%)$ & 0.056 \\
Fibrate & $130(25.8 \%)$ & $50(12.2 \%)$ & 0.058 \\
Ezetimibe & $36(8.2 \%)$ & $356(72.4 \%)$ & 0.345 \\
Beta Blocker & $351(69.6 \%)$ & $278(56.5 \%)$ & 0.424 \\
Nitrate & $271(54.0 \%)$ & $139(28.3 \%)$ & 0.372 \\
Ca Channel Blocker & & & 0.556 \\
\hline
\end{tabular}


Table 11. 12-Month Medication Use in PVD patients with BMS vs. DES

\begin{tabular}{lccc}
\hline & PVD + BMS & PVD + DES & p \\
& (N = 595) & (N = 542) & value \\
\hline Antiplatelet/Anticoagulant & $10(2.3 \%)$ & $2(0.5 \%)$ & 0.018 \\
No AP and no AC & $146(33.3 \%)$ & $57(12.7 \%)$ & $<0.001$ \\
Aspirin only & $13(3.0 \%)$ & $21(4.7 \%)$ & 0.183 \\
Thienopyridine only & $226(51.4 \%)$ & $360(79.8 \%)$ & $<0.001$ \\
Aspirin and Thienopyridine & $7(1.6 \%)$ & $3(0.7 \%)$ & 0.191 \\
Anticoagulation only & $37(8.5 \%)$ & $6(1.3 \%)$ & $<0.001$ \\
Single AP and AC & $22(5.0 \%)$ & $10(2.2 \%)$ & 0.026 \\
Triple therapy & $398(90.7 \%)$ & $407(90.9)$ & 0.923 \\
\hline Statin & $17(4.0 \%)$ & $8(1.9 \%)$ & 0.069 \\
Fibrate & $126(28.9 \%)$ & $58(13.8 \%)$ & 0.067 \\
Ezetimibe & $41(9.7 \%)$ & $298(67.1 \%)$ & 0.417 \\
Beta Blocker & $280(64.5 \%)$ & $149(32.3 \%)$ & 0.481 \\
Nitrate & $217(49.9 \%)$ & & 0.754 \\
Ca Channel Blocker & & & \\
\hline
\end{tabular}


Table 12. In-Hospital, 30-Day and 12-Month Outcomes in PVD patients with BMS vs. DES

\begin{tabular}{|c|c|c|c|}
\hline & $\begin{array}{c}\text { PVD + BMS } \\
(N=595)\end{array}$ & $\begin{array}{l}\text { PVD + DES } \\
(N=542)\end{array}$ & p value \\
\hline \multicolumn{4}{|l|}{ In-Hospital } \\
\hline MACE & $38(6.4 \%)$ & $15(2.8 \%)$ & 0.004 \\
\hline Death & $25(4.2 \%)$ & $10(1.9 \%)$ & 0.022 \\
\hline New or recurrent MI & $12(2.0 \%)$ & $1(0.2 \%)$ & 0.004 \\
\hline Repeat PCI & $1(0.2 \%)$ & $2(0.4 \%)$ & 0.507 \\
\hline CABGs & $4(0.7 \%)$ & $1(0.2 \%)$ & 0.215 \\
\hline New Renal Impairment & $13(2.2 \%)$ & $6(1.1 \%)$ & 0.158 \\
\hline Bleeding event & $22(3.7 \%)$ & $20(3.7 \%)$ & 0.995 \\
\hline Site of bleeding & & & 0.721 \\
\hline Retroperitoneal & $2(9.5 \%)$ & $2(10.0 \%)$ & \\
\hline Percutaneous entry site & $6(28.6 \%)$ & $8(40.0 \%)$ & \\
\hline Other & $13(61.9 \%)$ & $10(50.0 \%)$ & \\
\hline \multicolumn{4}{|l|}{ Vascular Complication } \\
\hline Access Site Occlusion & 0 & 0 & - \\
\hline Loss of distal pulse & $0(0.0 \%)$ & $1(0.2 \%)$ & 0.295 \\
\hline Peripheral Arterial Dissection & $4(0.7 \%)$ & $3(0.6 \%)$ & 0.158 \\
\hline AV Fistula & $1(0.2 \%)$ & $0(0.0 \%)$ & 0.340 \\
\hline Pseudoaneurysm & $0(0.0 \%)$ & $1(0.2 \%)$ & 0.295 \\
\hline \multicolumn{4}{|l|}{ 30-Days } \\
\hline MACE & $60(10.1 \%)$ & $26(4.8 \%)$ & 0.001 \\
\hline Death & $35(5.9 \%)$ & $14(2.6 \%)$ & 0.006 \\
\hline Cardiac cause & $25(71.4 \%)$ & $11(78.6 \%)$ & 0.609 \\
\hline
\end{tabular}




\begin{tabular}{lccc}
\hline Myocardial infarction & $25(4.2 \%)$ & $9(1.7 \%)$ & 0.012 \\
TVR & $13(2.2 \%)$ & $8(1.5 \%)$ & 0.375 \\
\hline 12-Months & $157(26.4 \%)$ & $105(19.4 \%)$ & 0.005 \\
MACE & $87(14.6 \%)$ & $46(8.5 \%)$ & 0.001 \\
Death & $44(50.6 \%)$ & $30(65.2 \%)$ & 0.106 \\
$\quad$ Cardiac cause & $61(10.3 \%)$ & $44(8.1 \%)$ & 0.214 \\
Myocardial infarction & $51(8.6 \%)$ & $46(8.5 \%)$ & 0.959 \\
TVR & & & \\
\hline
\end{tabular}

This article is protected by copyright. All rights reserved. 


\section{University Library}

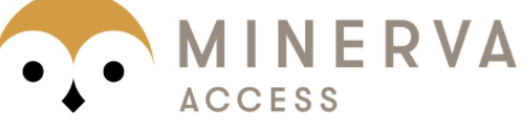

A gateway to Melbourne's research publications

Minerva Access is the Institutional Repository of The University of Melbourne

\section{Author/s:}

Ramzy, J;Andrianopoulos, N;Roberts, L;Duffy, SJ;Clark, D;Teh, AW;Ajani, AE;Reid, CM;Brennan, A;Freeman, M

Title:

Outcomes in patients with peripheral vascular disease following percutaneous coronary intervention

Date:

2019-10-01

Citation:

Ramzy, J., Andrianopoulos, N., Roberts, L., Duffy, S. J., Clark, D., Teh, A. W., Ajani, A. E., Reid, C. M., Brennan, A. \& Freeman, M. (2019). Outcomes in patients with peripheral vascular disease following percutaneous coronary intervention. CATHETERIZATION AND CARDIOVASCULAR INTERVENTIONS, 94 (4), pp.588-597. https://doi.org/10.1002/ ccd.28145.

Persistent Link:

http://hdl.handle.net/11343/285483 